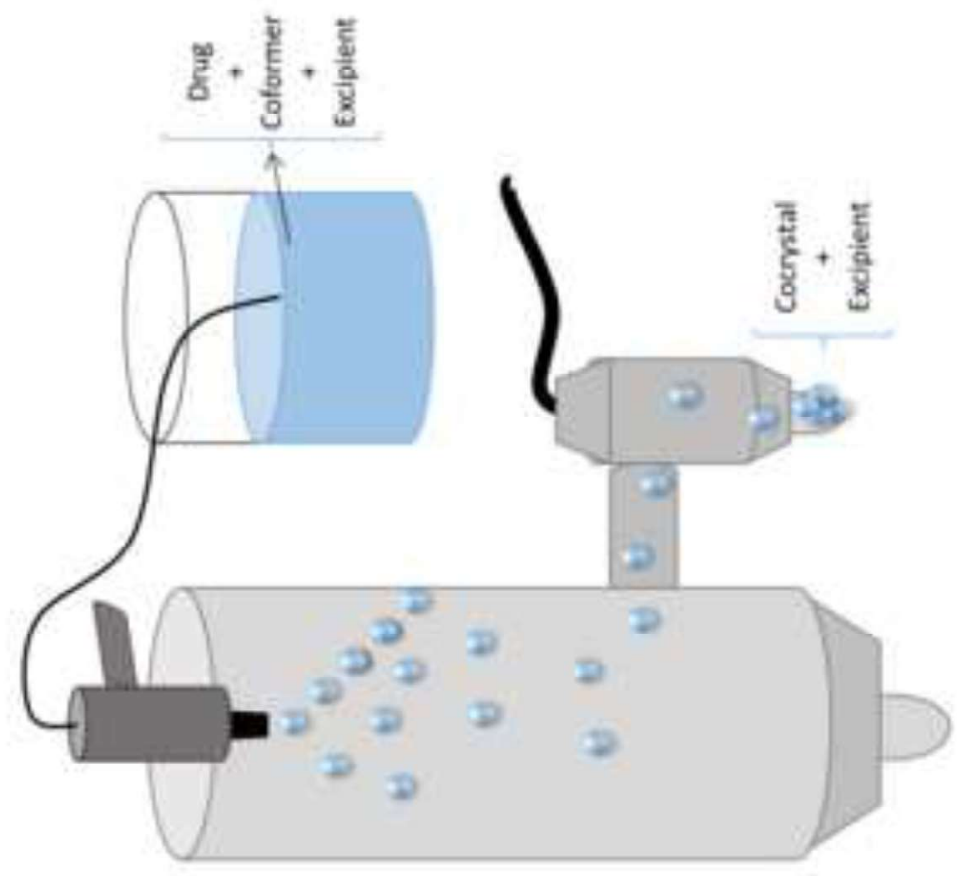

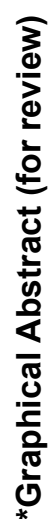


1

2

3

4

5

6

7

$8{ }^{\mathrm{b}}$ SSPC, Synthesis and Solid State Pharmaceutical Centre, Ireland.

9

10

11

12

13

14

15

16

17

18

19

20

21

22

23

24

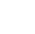

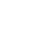

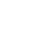

10

1

12

13

14

15

16

17

18

9 *Corresponding author:

$20 \quad$ Tel.: +35318961444

1 E-mail address: healyam@tcd.ie (A.M. Healy)

\title{
Production of Cocrystals in an Excipient Matrix by Spray Drying
}

\author{
David Walsh ${ }^{\mathrm{a}, \mathrm{b}}$, Dolores R. Serrano ${ }^{\mathrm{a}, \mathrm{b}}$, Zelalem Ayenew Worku ${ }^{\mathrm{a}, \mathrm{b}}$, Brid A. Norris ${ }^{\mathrm{a}, \mathrm{b}}$, Anne \\ Marie Healy $\mathrm{y}^{\mathrm{a}, \mathrm{b}^{*}}$
}

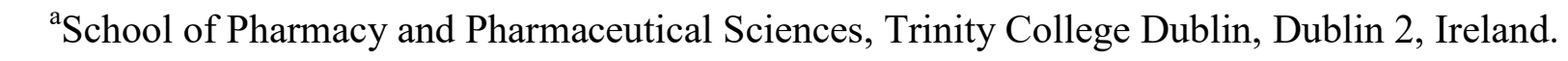




\section{Abstract}

26

27

Spray drying is a well-established scale-up technique for the production of cocrystals. However, to the best of our knowledge, the effect of introducing a third component into the feed solution during the spray drying process has never been investigated. Cocrystal formation in the presence of a third component by a one-step spray drying process has the potential to reduce the number of unit operations which are required to produce a final pharmaceutical product (e.g. by eliminating blending with excipient). Sulfadimidine (SDM), a poorly water soluble active pharmaceutical ingredient (API), and 4-aminosalicylic acid (4ASA), a hydrophilic molecule, were used as model drug and coformer respectively to form cocrystals by spray drying in the presence of a third component (excipient). The solubility of the cocrystal in the excipient was measured using a thermal analysis approach. Trends in measured solubility were in agreement with those determined by calculated Hansen Solubility Parameter (HSP) values. The ratio of cocrystal components to excipient was altered and cocrystal formation at different weight ratios was assessed. Cocrystal integrity was preserved when the cocrystal components were immiscible with the excipient, based on the difference in Hansen Solubility Parameters (HSP). For immiscible systems (difference in HSP $>9.6 \mathrm{MPa}^{0.5}$ ), cocrystal formation occurred even when the proportion of excipient was high $(90 \% \mathrm{w} / \mathrm{w})$. When the excipient was partly miscible with the cocrystal components, cocrystal formation was observed post spray drying, but crystalline API and coformer were also recovered in the processed powder. An amorphous dispersion was formed when the excipient was miscible with the cocrystal components even when the proportion of excipient used as low (10\% w/w excipient). For selected spray dried cocrystal-excipient systems an improvement in tableting characteristics was observed, relative to equivalent physical mixtures. 


\section{$51 \quad$ Keywords}

52 Spray drying, cocrystals, sulfadimidine, 4-aminosalicylic acid, Hansen Solubility Parameter, 53 industrial production intensification

54 


\section{Introduction}

It has been shown that the reason less than $1 \%$ of drug candidates make it to market is not only due to a lack of efficacy, safety or an unfavourable side effect profile, but also due to poor biopharmaceutical properties (Aakeröy Cb Fau - Aakeröy et al.; Cook et al., 2014). It has been suggested that drug discovery strategies, such as high throughput screening, are increasingly leading to lead candidates which have unfavourable physicochemical properties

64 (Lipinski et al., 2012). Many of these compounds have poor aqueous solubility, which can lead to a low dissolution rate (Hörter and Dressman, 2001). Over half of marketed drug products are formulated as salts to modify the physical properties of the active pharmaceutical ingredient (API) . However, a major limitation of this approach is the requirement of the API to possess a basic or acidic ionisable group. Pharmaceutical cocrystals offer an alternative to salt forms as a means of improving the solubility, dissolution and bioavailability of poorly water soluble drugs. Cocrystals of an API and coformer are formed by noncovalent, freely reversible interactions, and so the presence of an ionisable group is not a necessity. The solubility and dissolution rate of an API in a cocrystal are improved by lowering the lattice energy and/or increasing the solvent affinity (Thakuria et al., 2013). Cocrystallisation of an API can confer a number of advantages over other formulation strategies such as amorphisation. One of the major limitations of amorphous forms is the fact that they are thermodynamic unstable, making them prone to conversion to the lower energy crystalline forms (Hancock et al., 1995). 
Various methods exist to produce cocrystals. Common approaches include grinding and solution methods. However, a disadvantage of solution methods to produce cocrystals can be the formation of single component crystals when crystallised from an incongruently saturating solution (Qiao et al., 2011). Spray drying is commonly used to produce amorphous solid dispersions (Van den Mooter et al., 2001; Zhao et al., 2012) but also, in some instances, results in the formation of crystalline materials (Kumar et al., 2015). This technique has been shown to be a viable and scalable method to produce pure cocrystals from both congruent and incongruently saturating solutions. Carbamazepine-glutaric acid, theophylline-nicotinamide, urea-succinic acid and caffeine-glutaric acid all formed pure cocrystals when spray dried from an incongruently saturating solutions. Further to this, the urea-succinic acid 1:1 cocrystal was discovered and consistently generated in pure form by spray drying. Cocrystallisation of this system did not occur by slurry or reaction crystallisation methods (Alhalaweh and Velaga, 2010).

The approach of using Hansen Solubility Parameters (HSP) calculated using the group contribution method has enabled the prediction of solid-solid solubility of pharmaceutical materials (Greenhalgh et al.; Hancock et al., 1997). For drug-excipient combinations, a $\Delta \delta \mathbf{t}$ (i.e. difference in HSP) of less than $7.0 \mathrm{MPa}^{1 / 2}$ is considered to be indicative of significant miscibility, while a $\Delta \delta \mathbf{t}$ of greater than $10.0 \mathrm{MPa}^{1 / 2}$ denotes a lack of miscibility and limited ability to form glass solutions (Forster et al., 2001; Greenhalgh et al.).

97 Calculation of the HSP of drug and coformer and the difference in HSP values for the two components can be used as a tool to predict the success of cocrystal formation on spray drying. It has been shown that, in order for an API to form a cocrystal with a coformer, the two molecules must be miscible at a molecular level, with the difference in HSP being less than $7 \mathrm{MPa}^{0.5}$ (Mohammad et al., 2011). However, to the best of our knowledge, the effect on cocrystal formation of introducing a third (excipient) component into the feed solution during 
the spray drying process has never been investigated, nor has the relative differences in HSP between excipient and cocrystal components been probed in relation to success or otherwise of cocrystal formation on spray drying.

The hypothesis underlying this work is that a larger difference in HSP between the cocrystal components and the excipient will promote cocrystal formation during spray drying in the presence of a carrier excipient, as the cocrystal components will not be miscible with the excipient, and so will remain phase separated from the excipient but still interact with one another. In contrast, excipients which have a similar HSP to the cocrystal components may be miscible and may not allow for cocrystal formation to occur, rather there may be a high probability that an amorphous dispersion of individual coformer molecules, rather than a cocrystal suspension would form within the carrier.

114 The aim of this work was to investigate the impact of including a carrier excipient on cocrystal formation during the spray drying process. A range of pharmaceutical excipients were selected and co-spray dried with the cocrystal components. Solid state characterisation was performed as well as solubility studies of the cocrystal in the excipient using a thermal analysis approach. Dissolution studies were performed from constant surface area disks.

119 The feasibility of co-spray drying cocrystals and a third component, carrier excipient, in order

120 to reduce the number of unit processes to produce a final pharmaceutical product was investigated by compaction studies. 


\section{Materials}

Sulfadimidine (SDM), 4-aminosalicylic acid (4ASA), mannitol, chitosan (average molecular weight 50,000-190,000), glycine, polyvinyl alcohol (PVA) (average molecular weight 70,000-100,000), dextran (average molecular weight 68,800), hydroxypropyl methylcellulose (HPMC) (4,000 cP) and polyvinylpyrrolidone K15 (PVP) were purchased from SigmaAldrich (Ireland). Microcrystalline cellulose (MCC) Avicel ${ }^{\circledR}$ CL-611 was a gift from FMC Biopolymer, Belgium. Soluplus ${ }^{\circledR}$ was a gift from BASF, Germany. Inulin with an average degree of polymerisation of 11 (Fruitafit ${ }^{\circledR}$ HD) was a gift from Sensus, Netherlands. Ethanol was supplied by Corcoran Chemicals (Ireland). Water was purified and filtered using an Elix 3 connected to a Synergy UV system (Millipore, UK). All other chemicals used were of analytical grade.

\section{Methods}

\subsection{Preparation of cocrystals}

\section{Spray Drying}

A $1 \% \mathrm{w} / \mathrm{v}$ solution of SDM and 4-ASA was prepared using ethanol as solvent. The solution was sonicated to dissolve the cocrystal components completely. An equal volume of $1 \% \mathrm{w} / \mathrm{v}$ excipient aqueous solution (inulin, mannitol, glycine, PVA (heated to $60{ }^{\circ} \mathrm{C}$ ), HPMC, PVP and Soluplus) or suspension (MCC, chitosan and dextran) was added to the $1 \%$ solution of SDM and 4-ASA. The solution with the cocrystal components was mixed with the excipient solution/suspension prior to spray drying. The resultant solutions/suspensions were spray dried using a Büchi B-290 Mini Spray Dryer operating in the open mode. The solutions/suspensions were delivered to a 2-fluid atomization nozzle using a peristaltic pump at a pump speed of $30 \%(9-10 \mathrm{ml} / \mathrm{min})$ and the aspirator was operated at $35 \mathrm{~m}^{3} / \mathrm{hr}$. The 
148 flowmeter for the standard 2-fluid nozzle was set at $4 \mathrm{~cm}$, which is equivalent to 667 149 normlitres per hour $(\mathrm{Nl} / \mathrm{h})$ of gas flow at standard temperature and pressure conditions $150(\mathrm{p}=1013.25 \mathrm{mbar}$ and $\mathrm{T}=273.15 \mathrm{~K})$ (Büchi Labortechnik, 93001). The inlet temperature was 151 set at $105{ }^{\circ} \mathrm{C}$ (outlet temperature between $68-72{ }^{\circ} \mathrm{C}$ ) for the systems which contained 152 excipient in deionised water and $78^{\circ} \mathrm{C}$ (outlet temperature between $50-57^{\circ} \mathrm{C}$ ) for the spray drying of cocrystal in ethanol alone. Based on whether cocrystal formation occurred at this ratio of cocrystal component to excipient (i.e. $1: 1 \% \mathrm{w} / \mathrm{w}$ ), the ratio of cocrystal components to excipient was altered to assess the maximum ratio of excipient:cocrystal components which would allow cocrystal formation.

157 For comparison purposes, physical mixtures of cocrystal and excipients were prepared using an agate mortar and pestle.

\section{Solvent Evaporation}

Equimolar proportions of SDM and 4ASA were dissolved in $60 \mathrm{ml}$ of acetone to give a $0.01 \mathrm{M}$ solution and stirred until complete dissolution was achieved. The resulting solution was placed in a fumehood and allowed to evaporate for 72 hours (Serrano et al., 2016a).

\subsection{Solid State Characterisation}

\section{Powder X-Ray Diffraction}

Powder X-ray analysis was performed using a Miniflex II Rigaku diffractometer with Ni-

167 filtered $\mathrm{Cu} \mathrm{K \alpha}$ radiation (1.54 $\AA$ ). The tube voltage and tube current used were $30 \mathrm{kV}$ and 15 $\mathrm{mA}$, respectively. The PXRD patterns were recorded $(n=3)$ for 2 theta ranging from $5^{\circ}$ to $40^{\circ}$ at a step scan rate of $0.05^{\circ}$ per second. Rigaku Peak Integral software was used to determine peak intensity for each sample using the Sonneveld-Visser background edit procedure. 
172 DSC was performed using a Mettler Toledo DSC 821e instrument under nitrogen purge.

173 Powder samples (4-6 mg) were placed in aluminium pans $(40 \mu \mathrm{l})$, sealed, pierced to provide

174 three vent holes and heated at a rate of $10^{\circ} \mathrm{C} / \mathrm{min}$ in the temperature range of 25 to $250{ }^{\circ} \mathrm{C}$.

175 Temperature and enthalpy were calibrated using indium as standard. The DSC was controlled by Mettler Toledo STARe software (version 6.10) working on a Windows NT operating system. All reported temperatures refer to onset of melting.

\section{Solubility of Cocrystal in Excipient}

179 Physical mixtures of cocrystal and excipient were prepared by mixing in a pestle and mortar at different weight ratios. The melting enthalpy of the crystalline phase was determined by DSC (as described above) and plotted as a function of excipient weight fraction. The solubility of the cocrystal in excipient was determined by extrapolating the linear plot of the mass fraction against melting enthalpy to zero melting enthalpy, as previously described (Amharar et al., 2014). Annealing was not performed due to the thermal instability of 4-ASA.

\section{Attenuated Total Reflectance Fourier Transform Infrared Spectroscopy (ATR-FTIR)}

Infrared spectra were recorded on a PerkinElmer Spectrum 1 FT-IR Spectrometer equipped with a UATR and a ZnSe crystal accessory. Each spectrum was scanned in the range of 650$4000 \mathrm{~cm}^{-1}$ with a resolution of $4 \mathrm{~cm}^{-1}$. Data were evaluated using Spectrum v 5.0.1. software. Four scans of each sample were taken.

\section{Scanning Electron Microscope (SEM)}

The surface images of the samples were captured at various magnifications by SEM using a

Zeiss Supra Variable Pressure Field Emission Scanning Electron Microscope (Germany) equipped with a secondary electron detector at $5 \mathrm{kV}$. Samples were glued onto carbon tabs, 
mounted on to aluminium pin stubs and sputter-coated with gold/palladium under vacuum prior to analysis (Serrano et al., 2016b).

\subsection{Physical stability studies}

Spray dried samples $(100 \mathrm{mg})$ were placed in glass vials and stored in conditions of $25{ }^{\circ} \mathrm{C}$ and $60 \%$ relative humidity, with the required humidity provided by using a saturated solution of sodium bromide. After seven days, samples were removed and analysed by PXRD.

\subsection{Intrinsic dissolution studies}

The intrinsic dissolution studies of solid materials were performed using a Woods intrinsic dissolution apparatus (Elementec, Ireland). This allowed the dissolution to be measured from constant surface area discs. Discs were prepared by compressing the powder (200 mg) into compacts using a PerkinElmer hydraulic press with an $8 \mathrm{~mm}$ (diameter) punch and die set at a pressure of 3 tonnes for a $1 \mathrm{~min}$ dwell time. The dissolution studies were carried out in deionised water (volume: $900 \mathrm{~mL}$, temperature: $37{ }^{\circ} \mathrm{C}$ ) at a rotation speed of $100 \mathrm{rpm}$. Aliquots $(5 \mathrm{ml})$ were withdrawn with volume replacement at appropriate time intervals. Samples were filtered through $0.45 \mu \mathrm{m}$ filters and analysed for SDM and 4-ASA content by HPLC. The study was performed in triplicate. The intrinsic dissolution rate (IDR) was determined from the slope of the dissolution time profiles over the first 10 minutes. All dissolution studies were carried out for samples with a $50 \%(\mathrm{w} / \mathrm{w})$ ratio of excipient and cocrystal. At the end of the experiments, the discs were recovered, dried at ambient temperature and analysed by PXRD for process induced phase transformation.

Statistical analysis of dissolution profiles was performed using DDSolver (Zhang et al., 2010). Univariate ANOVA analysis and Similarity Factor $\left(f_{2}\right)$ analysis was performed to compare drug dissolution profiles (Yuksel et al., 2000). An $\mathrm{f}_{2}$ value between 50-100 indicates that dissolution profiles are similar. 


\subsection{High Performance Liquid Chromatography (HPLC)}

The concentration of SDM and 4-ASA in solution were determined as previously described (18) using an Alliance HPLC with a Waters 2695 Separations module system and Waters 2996 photodiode array detector. The mobile phase consisted of methanol and phosphate buffer $\mathrm{pH} 6.5$ in 40:60 (v/v) ratio. The buffer was prepared from a $50 \mathrm{mM}$ dipotassium phosphate solution adjusted to $\mathrm{pH} 6.5$ with phosphoric acid. The mobile phase was vacuum filtered through a $0.45 \mu \mathrm{m}$ membrane filter (Pall Supor ${ }^{\circledR} 0.45 \mu \mathrm{m}, 47 \mathrm{~mm}$ ) and bath sonicated for 5 min. Separation was performed on a Phenomenex Inertsil ODS (3) C18 column (150 mm length, diameter $4.6 \mathrm{~mm}$, particle size $5 \mu \mathrm{m}$ ) at a UV detection wavelength of $265 \mathrm{~nm}$. An injection volume of $20 \mu \mathrm{L}$ was used. The elution was carried out isocratically at ambient temperature with a flow rate of $1 \mathrm{~mL} / \mathrm{min}$. Elution times for 4-ASA and SDM were $1.9 \mathrm{~min}$ and 4.0 min respectively. Empower software was used for peak evaluation (Grossjohann et al., 2015).

\subsection{Compactability of cocrystals and cocrystal-polymer systems}

Tensile strength and ejection force of the co-spray dried systems and physical mixtures of cocrystal with MCC (50:50 w/w) or cocrytsal with inulin and MCC $(60: 20: 20 \mathrm{w} / \mathrm{w} / \mathrm{w})$ were investigated. Flat tablets $(\mathrm{n}=6,100 \mathrm{mg}$ ) were compressed using a Natoli NP-RD10 (Saint Charles, MO, USA) laboratory-scale single punch tablet press supplied with an Enerpac (Menomonee Falls, WI, USA) P-392 manual pump with a RC-104 hydraulic cylinder working in the range from 0 to 10 tonnes and standard 8-mm diameter punch and die tooling (I Holland Limited, UK). Compaction properties were quantified in terms of hardness achieved at the applied compaction pressure of $6 \mathrm{kN}$ (0.612 tonnes). The pressure was released immediately after the desired compression pressure was reached. Tablets were pushed out of the die using the bottom punch and ejection force was recorded. A set of 6 tablets was subjected to radial hardness testing using a Dr Schleuniger, Pharmatron model 6D 
243 tablet tester (Thun, Switzerland) (Serrano et al., 2016a). Tensile strength was calculated as

244 indicated in Equation 1:

$$
\sigma=\frac{2 * F}{\pi * D * H}
$$

246 in which $\sigma$ is the tensile strength, $F$ is the radial hardness, $D$ is the tablet diameter, and $H$ is

247 the tablet thickness. After compaction, it was monitored whether or not the tablet capped

248 under the applied pressure and if the breakage of the tablet occurred in a consistent manner.

249 The PXRD pattern of the formulation before and after compaction was compared.

\section{$250 \quad$ 3.7. Hansen Solubility Parameter Calculation}

251 Hansen solubility parameters were calculated from the chemical structures using the Van

252 Krevelen method (Van Krevelen and Te Nijenhuis, 2009). The weight average molecular

253 weights were used to determine solubility parameters for polymeric excipients (Scott, 1992).

254 The total HSP contribution was divided into three partial solubility parameters: dispersion

$255\left(\delta_{\mathrm{d}}\right)$, polar $\left(\delta_{\mathrm{p}}\right)$ and hydrogen bonding $\left(\delta_{\mathrm{h}}\right)$. The total solubility parameter was calculated as 256 indicated in Equations 2-5:

$$
\delta_{d}=\frac{\sum_{i=1}^{n} F_{d i}}{\sum_{i=1}^{n} v_{i}}
$$

$$
\delta_{\mathrm{t}}=\left(\delta_{\mathrm{d}}{ }^{2}+\delta_{\mathrm{p}}{ }^{2}+\delta_{\mathrm{h}}{ }^{2}\right)^{0.5}
$$

$$
\delta_{p}=\frac{\left(\sum_{i=1}^{n} F_{p i}^{2}\right)^{0.5}}{\sum_{i=1}^{n} v_{i}}
$$

$$
\delta_{h}=\left(\frac{\sum_{i=1}^{n} F_{h i}}{\sum_{i=1}^{n=1} v_{i}}\right)^{0.5}
$$


where $\mathrm{i}$ is the structural group within the molecule, $\mathrm{F}_{\mathrm{di}}$ is the group contribution of the dispersion forces, $F_{p i}$ is the group contribution of the polar forces, $F_{h i}$ is the group contribution of the hydrogen bonding forces, and $\mathrm{V}_{\mathrm{i}}$ is the group contribution of the molar volume (Mohammad et al., 2011).

\section{Results}

\subsection{Effect of the type and composition of excipient on cocrystal formation by spray drying}

\section{SDM/4-ASA cocrystal:excipient 50:50 (w/w)}

The polymorph II of the SDM:4-ASA cocrystal, the crystal structure of which has previously been determined by single crystal XRD (Grossjohann et al., 2015), was generated by spray drying. The X-ray diffraction pattern of SDM:4-ASA cocrystal and individual components are depicted in Figure 1, as well as the cocrystal prepared by slow solvent evaporation from acetone. DSC analysis of the cocrystals produced by solvent evaporation and spray drying showed a single endothermic peak, characteristic of cocrystal melting. The cocrystal produced by solvent evaporation had a higher melting point $\left(175.84 \pm 0.85^{\circ} \mathrm{C}\right)$ and a melting enthalpy $(239.15 \pm 6.84 \mathrm{~J} / \mathrm{g})$ compared to that produced by spray drying, which had a melting point of $170.08 \pm 0.23^{\circ} \mathrm{C}$ and a melting enthalpy of $216.52 \pm 3.69 \mathrm{~J} / \mathrm{g}$. This is in agreement with previously reported data (Grossjohann et al., 2015). This finding can be explained by the fact that rapid drying processes such as spray drying are likely to induce crystal lattice imperfections such as point defects, line defects and plane defects, which can affect the thermal properties of the spray dried product (Corrigan, 1995).

PXRD demonstrated cocrystal formation was preserved when cocrystal components were spray dried in the presence of inulin, MCC, dextran and mannitol at a $50 \%(\mathrm{w} / \mathrm{w})$ ratio of 
cocrystal components to $50 \%(\mathrm{w} / \mathrm{w})$ of excipient. PXRD analyses showed that the same diffraction peaks were present when compared to the spray dried cocrystal. Characteristic diffractions peaks of the cocrystal are observed at $11.9^{\circ}, 13.65^{\circ}, 20.25^{\circ}$ and $24.4^{\circ} 2 \theta$ (Serrano et al., 2016a). It would be expected that cocrystal formation would occur in the presence of a suspended excipient (which was the case for MCC, chitosan and dextran), as the cocrystal components in solution would be phase separated from the excipient in suspension. Extra diffraction peaks were present for the cocrystal in mannitol system which were attributed to mannitol (both alpha and delta polymorphs). Characteristic peaks of delta mannitol are present at $9.75^{\circ}$ and $25.2^{\circ} 2 \theta$, while characteristic alpha mannitol peaks are observed at $17.3^{\circ}$ and $33.2^{\circ} 2 \theta$. Spray drying of mannitol and lysosome has previously been shown to produce a system containing a mixture of mannitol polymorphs, and both beta and delta polymorphs of mannitol were observed (Hulse et al., 2009). However, the intensity of the diffraction peaks was decreased for the co-spray dried cocrystal in excipient system when compared to a physical mixture of the spray dried cocrystal and excipient, probably due to the interaction between the cocrystal components and the excipient, and partial amorphisation of cocrystal within the excipient matrix. Reduction in peak intensity may also be attributed to crystal imperfections and/or the preferred orientation effect (Grant and York, 1986). The observed decrease in intensity varied for each excipient used. PXRD analyses of of physical mixtures of cocrystal and excipient are shown in Figure S1, Supplementary material.

The melting enthalpy associated with the co-spray dried cocrystal in inulin system was 91.81 $\pm 2.62 \mathrm{~J} / \mathrm{g}$, compared with a value of $98.7 \pm 5.45 \mathrm{~J} / \mathrm{g}$ for a physical mixture of the spray dried cocrystal and inulin. The co-spray dried dextran in cocrystal system showed an enthalpy of $99.11 \pm 5.4 \mathrm{~J} / \mathrm{g}$, compared to a value of $103.21 \pm 9.13 \mathrm{~J} / \mathrm{g}$ for the physical mixture of dextran and spray dried cocrystal. The excipient which showed the largest difference in enthalpy between the co-spray dried system and the physical mixture was MCC, with values of 83.52 
$310 \pm 4.23 \mathrm{~J} / \mathrm{g}$ and $101.02 \pm 9.59 \mathrm{~J} / \mathrm{g}$ respectively. In all cases, the only endothermic event was

311 attributed to the melting of the cocrystal, and no exothermic events were observed. It was not

312 possible to accurately measure the enthalpy of melting for the cocrystal when mannitol was

313 used as an excipient. Mannitol melted at $165.46 \pm 0.47^{\circ} \mathrm{C}$, which overlapped with the melting

314 of the cocrystal. Based on the DSC analyses, the relative crystallinities of the co-spray dried systems compared to the physical mixtures were $93.02 \%, 96.03 \%$ and $82.68 \%$ for the systems containing inulin, dextran and MCC respectively. The co-spray dried systems had a similar melting temperature as the physical mixture of cocrystal and excipient for all systems with the exception of MCC, where a significant melting point depression was seen for the cospray dried formulation when compared to the physical mixture. DSC analyses of the physical mixtures are shown in Figure S2, Supplemental material.

Bragg diffraction peaks attributable to the cocrystal, as well as the individual components (API and coformer), were observed when cocrystal components were spray dried in the presence of PVA, glycine and chitosan at a $50: 50 \% \mathrm{w} / \mathrm{w}$ ratio. Characteristic diffraction peaks of glycine were also present in that particular system (Figure S3, Supplementary material). An amorphous solid dispersion was produced when cocrystal components were spray dried in the presence of Soluplus, HPMC and PVP at the 50/50\% (w/w) ratio (Figure S4, Supplementary material).

Based on the calculated HSP, inulin, MCC, mannitol, chitosan and dextran are immiscible with the cocrystal components with a difference in HSP between the excipient and cocrystal ranging from 9.6 $\mathrm{MPa}^{0.5}-18.6 \mathrm{MPa}^{0.5}$ (Table 1). All of these spray dried systems, with the exception of chitosan, resulted in the formation of a cocrystal and there was no evidence of other (individual API or coformer) components present by PXRD. Characteristic diffraction peaks of the cocrystal and SDM were observed for the spray dried system containing 
chitosan. As chitosan is a basic polymer, there may be an interaction with the acidic

336 The differences in HSP between PVA and glycine and the cocrystal are $4.9 \mathrm{MPa}^{0.5}$ and 6.6

$\mathrm{MPa}^{0.5}$, respectively which can explain the presence of diffraction peaks of both the cocrystal and the individual components, due to the partial miscibility of the cocrystal components within these excipients. It may be hypothesised that the interaction of the excipient with the cocrystal components can result in the formation of an amorphous dispersion. The diffraction peaks observed may be as a result of the rapid crystallisation of a binary, ternary or single component amorphous domains. The crystallisation of materials by spray drying is thought to be a two stage process, with material transforming from the liquid to an amorphous phase first, and then from the amorphous phase to a crystalline phase (Chiou and Langrish, 2008) higher miscibility of the cocrystal components in these excipients.

\subsection{Effect of different ratios of excipient on cocrystal formation during spray drying}

\section{PVP, Soluplus and HPMC}

The ratio of cocrystal components to excipient was altered to assess whether the HSP difference reflected the ratio at which a cocrystal would form when co-spray dried with an excipient. PVP, Soluplus and HPMC were chosen and different cocrystal:excipient weight ratios $(75: 25,80: 20,90: 10)$ investigated.

At the lowest ratio of excipient $(10 \% \mathrm{w} / \mathrm{w})$, the cocrystal was formed when PVP and Soluplus were the excipients used. However, an amorphous dispersion was formed in the case of 
HPMC (data not shown). It has previously been determined that viscous polymers can inhibit the crystallisation process. The fast evaporation of solvent which occurs during the drying process can lead to a rapid viscosity increase and permit kinetic trapping of the cocrystal components in the excipient matrix as an amorphous form or disordered system (Paudel et al., 2013). As the HPMC solution has a higher viscosity than the PVP and Soluplus solutions (data not shown), both the higher viscosity and the lower difference in HSP between the cocrystal components and HPMC may contribute to the formation of an amorphous dispersion.

For PVP and Soluplus, cocrystal formation was observed when excipients were co-spray dried at a ratio of $80: 20(\mathrm{w} / \mathrm{w})$ cocrystal components to excipient (Figure $2 \mathrm{i}$, iii). When the ratio was altered to $75: 25(\mathrm{w} / \mathrm{w})$ cocrystal components to excipient, an amorphous dispersion was formed in the case of both excipients. The three co-spray dried PVP and Soluplus systems at different ratios were then stressed under conditions of $25^{\circ} \mathrm{C}$ and $60 \%$ relative humidity $(\mathrm{RH})$ for one week. An increased intensity of the Bragg peaks was observed in those co-spray dried systems containing $80 \%$ and $90 \%$ cocrystal. Co-spray dried cocrystal components and PVP at a $75 \%(\mathrm{w} / \mathrm{w})$ cocrystal components to $25 \%(\mathrm{w} / \mathrm{w})$ ratio crystallised from an amorphous dispersion to the metastable polymorph II cocrystal (Grossjohann et al., 2015) under these conditions. Peaks attributable to individual components or to the form I cocrystal (22) were not observed. In contrast, when the 75:25 (w/w) cocrystal components:Soluplus system was stressed, diffraction peaks attributable to both the form II and more stable form I cocrystal were present (Figure 2ii, iv). When the cocrystal alone (which presents as form II) was stressed under the same conditions, a polymorphic transition to the form I cocrystal was not observed, suggesting that stressing co-spray dried cocrystal: Soluplus (75:25\% w:w) from the amorphous state results in a metastable form II. 
Diffraction peaks attributable to both the cocrystal and individual components were seen when chitosan $(50 \% \mathrm{w} / \mathrm{w})$ was co-spray dried with cocrystal components $(50 \% \mathrm{w} / \mathrm{w})$. This ratio was altered to determine the maximum ratio at which cocrystal formation will occur without the presence of individual components. Cocrystal formation occurred when $10 \%$, $20 \%$ and $25 \%(\mathrm{w} / \mathrm{w})$ chitosan was co-spray dried with the cocrystal components. When $30 \%$ of chitosan was used, cocrystal as well as the peaks of individual components were observed, probably due to the interaction between the chitosan and the 4ASA, as previously commented. DSC thermograms showed that the melting temperature of the co-spray dried system with chitosan varied between 164 to $167^{\circ} \mathrm{C}$ (Figure 3 ) .

A cocrystal was formed in the presence of MCC when the cocrystal components $(50 \% \mathrm{w} / \mathrm{w})$ were co-spray dried with $\mathrm{MCC}(50 \% \mathrm{w} / \mathrm{w})$. As a cocrystal formed at this ratio, the amount of MCC relative to cocrystal components was increased to assess the maximum ratio at which cocrystal formation would occur. Cocrystal formation was observed up to a 30:70, cocrystal:MCC weight ratio. A reduction in intensity of Bragg peaks attributable to the cocrystal was seen when the ratio of MCC to cocrystal components was increased (Figure 4i).

401 The diffraction pattern was devoid of characteristic Bragg peaks of the individual components. The melting point depression of the cocrystal with increasing MCC composition suggests the formation of a more imperfect crystalline form of the cocrystal when higher ratios of MCC are used. A broader melting peak can be attributed to imperfect crystalline form (Figure 4iii). After stressing at $25^{\circ} \mathrm{C}$ and $60 \% \mathrm{RH}$ for seven days, characteristic Bragg 
peaks of the cocrystal were observed even at the lowest ratio (cocrystal: MCC, 20:80) (Figure 4ii).

\subsection{Morphology}

410

Spray drying resulted in cocrystal microspheres between 1-10 $\mu \mathrm{m}$ (Figure 5). Microparticle surface and morphology was dependent on the excipient used, but also on the excipientcocrystal ratio. In those systems where the cocrystal was formed, microspheres exhibited rough surfaces with embedded crystals at the surface (Figure 5a-d) whereas, in those systems where an amorphous solid dispersion was formed, microspheres exhibited smooth surfaces (for example with PVP at 50\%). When the ratio of PVP was reduced to $10 \%$, cocrystal formation occurred and microspheres with rough surfaces were observed (Figure 5f).

\subsection{ATR-FTIR}

The H-bonding interaction between the cocrystal and the excipients were analysed by ATRFTIR (Figure 6). Distinctive bands in the higher frequency range were observed for the single components. Asymmetric and symmetric stretching bands of $-\mathrm{NH}_{2}$ of 4ASA were observed at $3493 \mathrm{~cm}^{-1}$ and $3386 \mathrm{~cm}^{-1}$. SDM displays asymmetric and symmetric stretching bands of the $\mathrm{NH}_{2}$ group at $3441 \mathrm{~cm}^{-1}$ and $3339 \mathrm{~cm}^{-1}$ respectively. The sulphonamide $\mathrm{NH}$ group shows a stretching band at $3235 \mathrm{~cm}^{-1}$. The molecular interaction through hydrogen bond formation between SDM and 4ASA spray dried cocrystal was characterised by: i) two broad bands, one at $3482 \mathrm{~cm}^{-1}$ and one at $3372 \mathrm{~cm}^{-1}$ with a shoulder attributable to the N-H stretching of the

$427 \mathrm{NH}_{2}$ amine group of 4ASA which were shifted towards lower wavenumbers from $3493 \mathrm{~cm}^{-1}$ 428 and $3386 \mathrm{~cm}^{-1}$ and ii) sulfone $\left(-\mathrm{SO}_{2}\right)$ stretching in SDM and $-\mathrm{OH}$ deformation in 4ASA at $4291315 \mathrm{~cm}^{-1}$ and $1275 \mathrm{~cm}^{-1}$, respectively (Grossjohann et al., 2015). The same bands were seen 
430 for both the spray dried cocrystal alone and the co-spray dried systems (containing inulin,

431

432

433

434

435

436

437

438

439

440

441

442

444

445

446

447

448

449

450

451

452

453 mannitol, MCC and dextran), indicating no interaction between the cocrystal and the excipient during spray drying. Hydrogen bonding attributable to cocrystal formation is not seen when PVP and Soluplus were co-spray dried with the cocrystal components at the 50:50 $\%$ w/w ratio. In Figure 5, the co-spray dried system with inulin is illustrated. Co-spray dried systems with dextran, MCC, mannitol, PVP and Solulpus at the 50\% (w/w) ratio are presented in Figure S5-S9, supplementary material).

\subsection{Solubility of cocrystal in excipient}

We hypothesised that cocrystal formation occurs in the presence of an excipient when the single components are not miscible with the excipient, as determined by the difference in HSP between the components and excipient. In order to correlate the difference in HSP with the miscibility of the cocrystal with the excipient matrix, the solubility of the spray dried cocrystal and the individual cocrystal components in the amorphous excipients (inulin, MCC, dextran, chitosan, PVA, PVP, Soluplus and HPMC) was determined by the zero melting enthalpy extrapolation method (Amharar et al., 2014). The solubility of the cocrystal in inulin, MCC and dextran was 3.69\% w/w, 3.85\% w/w and 3.83\% w/w, respectively, which was relatively low (Figure 7). These results were in agreement with the differences in HSP between the cocrystal and excipient of $18.6 \mathrm{MPa}^{0.5}, 12.5 \mathrm{MPa}^{0.5}$ and $9.6 \mathrm{MPa}^{0.5}$ respectively, indicating that the formation of the cocrystal at higher excipient ratios is likely to happen. The solubility of cocrystal in chitosan was determined to be $3.23 \%$. This value is in agreement with the calculated HSP difference of $11.2 \mathrm{MPa}^{0.5}$ between the cocrystal and chitosan (Figure 7). However, a cocrystal only formed at low ratios of chitosan possibly due to the interaction between basic chitosan and acidic 4ASA. 
454 The solubility of the cocrystal in PVA was $13.74 \% \mathrm{w} / \mathrm{w}$ (Figure 8 ) and the difference in HSP

455 between the cocrystal and PVA was $4.9 \mathrm{MPa}^{0.5}$. Cocrystal solubility in PVP, Soluplus and

456

457

458

459

460

461

462

463

464

465

466

467

468

469

470

471

472

473

474

475

476

477 HPMC was much higher, 24.43\%w/w, 25.21\% w/w and $18.77 \% \mathrm{w} / \mathrm{w}$ respectively (Figure 8).

These values were also in agreement with the differences in HSP between the cocrystal and excipient, indicating higher miscibility between the cocrystal and the excipient justifying why cocrystal formation only occurred when a low ratio of excipient was used. Similar solubility values between the single components and the excipients were observed (Values in Table 2) (Figure S10 - S12, Supplementary material).

\subsection{Dissolution Studies}

Dissolution of SDM and 4-ASA from the cocrystal started incongruently over the first $10 \mathrm{~min}$ and became congruent subsequently (Figure S13, Supplementary material). During spray drying, 4ASA can partially sublime, resulting in a mass loss of 4ASA, as previously reported (Grossjohann et al., 2015). HPLC analysis of the spray dried cocrystal showed $3.5 \%$ less molar amount of 4ASA in the final spray dried formulation. This resulted in an excess of $\mathrm{SDM}$ in the spray dried product which can transform to the amorphous state upon spray drying (Caron et al., 2011). Once the excess amorphous SDM crystallised, dissolution became congruent.

No statistically significant differences in the $f_{2}$ value were found among the dissolution profiles of the co-spray dried systems $(50: 50 \% \mathrm{w} / \mathrm{w}$ ratio) with inulin, mannitol or dextran (Figure 9). Dissolution from a constant surface area could not be tested when MCC was used as an excipient since, due to the disintegrant properties of $\mathrm{MCC}$, the disk quickly disintegrated. No differences were found between the intrinsic dissolution rates of the three co-spray dried systems (Table 3). Therefore, it was concluded that the excipient used had no impact on the dissolution of the cocrystal from the co-spray dried system. 
478 After dissolution, the compacts were dried and analysed by PXRD for surface changes. A

479 polymorphic transformation from the form II to form I was observed from the co-spray dried system with mannitol. In contrast, no polymorphic transformation was seen when dissolution studies were performed with inulin and dextran (Figures S14 - S16, Supplementary material). The compacts were smooth and homogenous before dissolution. After dissolution, the surface was observedo be pitted due to the different dissolution rates of the excipient and cocrystal.

\subsection{Compactability of spray dried cocrystal:excipient systems}

As a proof of concept, the feasibility of co-spray dried systems to reduce the number of unit processes to produce a final pharmaceutical product was investigated by compaction studies. As MCC is commonly used as a tablet filler due to its excellent compression properties (David and Augsburger, 1977), the compactability of the co-spray dried system with MCC $(50 \% \mathrm{w} / \mathrm{w})$ and its corresponding physical mixture were assessed. Including more than one excipient in the feed solution/suspension may allow for a blending step to be omitted, going directly from a spray drying process to a direct compression. For this reason, the compaction properties of a co-spray dried system containing $60 \% \mathrm{w} / \mathrm{w}$ cocrystal, $20 \% \mathrm{w} / \mathrm{w}$ inulin and 20 $\%$ w/w MCC was also assessed, along with a physical mixture with identical composition. It has previously been reported that the SDM:4ASA cocrystal produced by spray drying is less prone to capping than the cocrystal produced by solvent evaporation (Serrano et al., 2016a). For the MCC systems, both the co-spray dried system and physical mixture produced tablets with similar tensile strengths. A significant difference in ejection force was observed however, with the co-spray dried system requiring a 5-fold lower force to eject the tablets (Figure 10). No capped tablets were observed for both the co-spray dried system and the physical mixture. PXRD analyses was performed to assess possible alteration of the crystal structure during the tabletting process. While an increase in Bragg peak intensity was 
503 observed for the co-spray dried system after compaction, no deformation induced phase 504 transformation changes were observed (Figure S17, Supplementary material). For the system 505 containing both MCC and inulin, the co-spray dried system showed no tendency to capping 506 during compaction. Two capped tablets were observed for the physical mixture. These two 507 tablets were not tested further. Two extra tablets were made and tested. No differences were 508 observed in tensile strength between the co-spray dried system and the physical mixtures. 509 However, a significantly lower ejection force (19-fold) was observed for the co-spray dried 510 system (Figure 10), suggesting that the compaction properties of the co-spray dried system 511 were notably improved, due to less sticking characteristics. Possible alteration of the 512 cocrystal structure was evaluated by PXRD analysis before and after the compaction. No

513 deformation induced phase transformation changes were observed (Figure S18, 514 Supplementary material).

\section{5. Discussion}

517 This study has demonstrated the feasibility of cocrystal formation and inclusion within an excipient matrix, through the process of co-spray drying. PXRD and DSC analysis for the cocrystal-in-excipient systems were consistent with those of the cocrystal produced by solvent evaporation, indicating that cocrystal formation still occurred when the cocrystal was co-spray dried with some of the excipients included in this study.

522 Differences in DSC results were noted between the cocrystal-in-excipient systems and the 523 corresponding physical mixtures; it was found that the heat of fusion was lowered (and the melting temperature depressed when higher ratios of excipient were used) for the co-spray 
drying process induced some level of amorphisation of the cocrystal, without fully impeding cocrystal formation.

528

Previously, it has been determined that a difference in HSP of less than $7 \mathrm{MPa}^{0.5}$ indicates

529

530

547 Where $\triangle \mathrm{HSP}$ is the difference in HSP between the cocrystal and the excipient, $\mathrm{F}_{\mathrm{e}}$ is the

that materials are miscible. This theory has been utilised to predict cocrystal formation, whereby drug and coformer with $\Delta \mathrm{HSP}<7 \mathrm{MPa}^{0.5}$ were shown to be likely to form a cocrystal due to their miscibility. In this study, the same principle was applied to predict cocrystal formation in the presence of a carrier excipient. However, in this case it was anticipated that the closer the value of HSP for the cocrystal and carrier excipient, the less likely cocrystal formation would be because the carrier excipient would be miscible in the cocrystal and thus prevent cocrystal formation. The findings from the study showed that a clear correlation exists between the HSP difference between the cocrystal and carrier excipient and the likelihood of cocrystal formation occurring. It can be deduced that $\Delta \mathrm{HSP}>$ 9.6 $\mathrm{MPa}^{0.5}$ for the cocrystal and carrier excipient leads to formation of the cocrystal when it is co-spray dried with the carrier excipient. $\triangle \mathrm{HSP}<9.6 \mathrm{MPa}^{0.5}$ for the cocrystal and carrier excipient results in either a completely amorphous form following co-spray drying, or cocrystal with traces of the individual components (API, coformer) of the cocrystal.

The ratio of excipient:cocrystal had a major impact on cocrystal formation as well as the overall miscibility between the cocrystal and the excipient. In order to get a deeper insight into the process, a parameter to predict cocrystal formation (CFP) was calculated using Equation 6:

$$
C F P=\frac{\triangle H S P}{F e * S}
$$
excipient fraction and $\mathrm{S}$ is the measured solubility of the cocrystal within the excipient matrix. Based on the CFP calculated values and the experimental results (Table 4), it can be 
550 concluded that for those systems with a CFP value $>10$, there is a high probability of 551 cocrystal formation, while values below 1 indicate that there is a high probability of co-

552 amorphous systems forming. Some exceptions were found, such as chitosan, probably due to 553 its basic behaviour and interaction in solution with the coformer decreasing the H-bonding 554 with SDM.

555 For those co-spray dried systems that allowed cocrystal formation, FTIR revealed no 556 interaction between the cocrystal and the excipient. Also, intrinsic dissolution studies showed 557 no differences in the SDM release rate among the different excipients suggesting that the 558 release of SDM was determined by the cocrystal itself. Preliminary studies on process 559 intensification showed that co-spray dried systems had better compaction properties than 560 physical mixtures, suggesting that a secondary excipient blending step might be avoided.

\section{Conclusions}

562 This work demonstrates that the introduction of a third component into the feed 563 solution/suspension prior to spray drying can result in a cocrystal embedded in excipient 564 matrix. Cocrystal formation can also occur when more than one excipient is added to the spray drying feed solution/suspension. The difference in HSP between the cocrystal components and the excipient can be used as a general parameter to predict if cocrystal

567 formation will occur. However, as was seen when the cocrystal components were co-spray dried with chitosan, other factors such as the acidic/basic nature of the excipient can 569 influence whether cocrystal formation can occur. The difference in HSP can also be used to 570 predict the ratio at which a cocrystal can form when co-spray dried with an excipient. Cospray drying an excipient with the cocrystal components can result in cocrystal formation, regardless of the crystalline or amorphous nature of the excipient. As spray drying is a

573 scalable unit operation used in the pharmaceutical industry, co-spray drying with an excipient 
can reduce the number of unit operations required to produce a final pharmaceutical product, as a separate blending step of the cocrystal and excipient could be avoided.

\section{Acknowledges}

This publication has emanated from research supported in part by a research grant from Science Foundation Ireland (SFI) [Grant Number 12/RC/2275] and is co-funded under the European Regional Development Fund.

\section{References}

(a) Handbook of Pharmaceutical Salts: Properties, Selection and Use; Stahl, P. H.; Wermuth, C. G. Eds.; Verlag Helvetica Chimica Acta: Zu“rich,2002.(b)Bighley,L.D.;Berge,S.M.;Monkhouse,D.C. Salt forms of drugs and absorption. In Encyclopedia of Pharmaceutical Technology; Swarbick, J.; Boylan, J. C. Eds; Marcel Dekker, Inc.: New York, 1996; Vol. 13, pp 453-499. (c) Gu, C. H.; Grant, D. J. W. Handbook of Experimental Pharmacology: Stereochemical Aspects of Drug Action and Disposition; Eichelbaum, M.; Testa, B.; Somogyi, A. Eds.; Springer: Berlin, 2003.

Aakeröy Cb Fau - Aakeröy, C.B., Forbes S Fau - Forbes, S., Desper J Fau - Desper, J., Using Cocrystals To Systematically Modulate Aqueous Solubility and Melting Behavior of an Anticancer Drug. Alhalaweh, A., Velaga, S.P., 2010. Formation of Cocrystals from Stoichiometric Solutions of Incongruently Saturating Systems by Spray Drying. Crystal Growth \& Design 10, 3302-3305. Amharar, Y., Curtin, V., Gallagher, K.H., Healy, A.M., 2014. Solubility of crystalline organic compounds in high and low molecular weight amorphous matrices above and below the glass transition by zero enthalpy extrapolation. International Journal of Pharmaceutics 472, 241-247. Antoniou, E., Themistou, E., Sarkar, B., Tsianou, M., Alexandridis, P., 2010. Structure and dynamics of dextran in binary mixtures of a good and a bad solvent. Colloid and Polymer Science 288, 1301-1312. Caron, V., Tajber, L., Corrigan, O.I., Healy, A.M., 2011. A Comparison of Spray Drying and Milling in the Production of Amorphous Dispersions of Sulfathiazole/Polyvinylpyrrolidone and Sulfadimidine/Polyvinylpyrrolidone. Molecular Pharmaceutics 8, 532-542. Chiou, D., Langrish, T.A.G., 2008. A comparison of crystallisation approaches in spray drying. Journal of Food Engineering 88, 177-185.

Cook, D., Brown, D., Alexander, R., March, R., Morgan, P., Satterthwaite, G., Pangalos, M.N., 2014. Lessons learned from the fate of AstraZeneca's drug pipeline: a five-dimensional framework. Nat Rev Drug Discov 13, 419-431.

Corrigan, O.I., 1995. Thermal analysis of spray dried products. Thermochimica Acta 248, 245-258. David, S.T., Augsburger, L.L., 1977. Plastic flow during compression of directly compressible fillers and its effect on tablet strength. Journal of pharmaceutical sciences 66, 155-159. Forster, A., Hempenstall, J., Tucker, I., Rades, T., 2001. Selection of excipients for melt extrusion with two poorly water-soluble drugs by solubility parameter calculation and thermal analysis. International Journal of Pharmaceutics 226, 147-161. 
613 Grant, D.J.W., York, P., 1986. Entropy of processing: a new quantity for comparing the solid state disorder of pharmaceutical materials. International Journal of Pharmaceutics 30, 161-180. Greenhalgh, D.J., Williams, A.C., Timmins, P., York, P., Solubility parameters as predictors of miscibility in solid dispersions. Journal of Pharmaceutical Sciences 88, 1182-1190. Grossjohann, C., Serrano, D.R., Paluch, K.J., O'Connell, P., Vella-Zarb, L., Manesiotis, P., McCabe, T., Cocrystals: Solid-State Characterization and Physicochemical Properties. Journal of Pharmaceutical Sciences 104, 1385-1398.

Hancock, B.C., Shamblin, S.L., Zografi, G., 1995. Molecular mobility of amorphous pharmaceutical solids below their glass transition temperatures. Pharmaceutical research 12, 799-806.

Hancock, B.C., York, P., Rowe, R.C., 1997. The use of solubility parameters in pharmaceutical dosage form design. International Journal of Pharmaceutics 148, 1-21.

Hörter, D., Dressman, J.B., 2001. Influence of physicochemical properties on dissolution of drugs in the gastrointestinal tract1. Advanced Drug Delivery Reviews 46, 75-87.

Hulse, W.L., Forbes, R.T., Bonner, M.C., Getrost, M., 2009. Influence of protein on mannitol polymorphic form produced during co-spray drying. International Journal of Pharmaceutics 382, 6772.

Kumar, S., Shen, J., Zolnik, B., Sadrieh, N., Burgess, D.J., 2015. Optimization and dissolution performance of spray-dried naproxen nano-crystals. International Journal of Pharmaceutics 486, 159-166.

Lipinski, C.A., Lombardo, F., Dominy, B.W., Feeney, P.J., 2012. Experimental and computational approaches to estimate solubility and permeability in drug discovery and development settings. Advanced drug delivery reviews 64, 4-17.

Mohammad, M.A., Alhalaweh, A., Velaga, S.P., 2011. Hansen solubility parameter as a tool to predict cocrystal formation. International Journal of Pharmaceutics 407, 63-71.

Paudel, A., Worku, Z.A., Meeus, J., Guns, S., Van den Mooter, G., 2013. Manufacturing of solid dispersions of poorly water soluble drugs by spray drying: Formulation and process considerations. International Journal of Pharmaceutics 453, 253-284.

Qiao, N., Li, M., Schlindwein, W., Malek, N., Davies, A., Trappitt, G., 2011. Pharmaceutical cocrystals: An overview. International Journal of Pharmaceutics 419, 1-11.

Ravindra, R., Krovvidi, K.R., Khan, A.A., 1998. Solubility parameter of chitin and chitosan. Carbohydrate Polymers 36, 121-127.

Rowe, R.C., 1988. Interaction of lubricants with microcrystalline cellulose and anhydrous lactose - a solubility parameter approach. International Journal of Pharmaceutics 41, 223-226.

Scott, G., 1992. Properties of polymers. Their correlation with chemical structure; their numerical estimation and prediction from additive group contributions. Endeavour 16, 97-98.

Serrano, D.R., O'Connell, P., Paluch, K.J., Walsh, D., Healy, A.M., 2016a. Cocrystal habit engineering to improve drug dissolution and alter derived powder properties. J Pharm Pharmacol 68, 665-677. Serrano, D.R., Persoons, T., D'Arcy, D.M., Galiana, C., Dea-Ayuela, M.A., Healy, A.M., 2016b. Modelling and shadowgraph imaging of cocrystal dissolution and assessment of in vitro antimicrobial activity for sulfadimidine/4-aminosalicylic acid cocrystals. Eur J Pharm Sci 89, 125-136.

Thakuria, R., Delori, A., Jones, W., Lipert, M.P., Roy, L., Rodríguez-Hornedo, N., 2013. Pharmaceutical cocrystals and poorly soluble drugs. International Journal of Pharmaceutics 453, 101-125.

Van den Mooter, G., Wuyts, M., Blaton, N., Busson, R., Grobet, P., Augustijns, P., Kinget, R., 2001. Physical stabilisation of amorphous ketoconazole in solid dispersions with polyvinylpyrrolidone $\mathrm{K} 25$. European Journal of Pharmaceutical Sciences 12, 261-269.

Van Krevelen, D.W., Te Nijenhuis, K., 2009. Chapter 7 - Cohesive Properties and Solubility, Properties of Polymers (Fourth Edition). Elsevier, Amsterdam, pp. 189-227.

Yuksel, N., Kanık, A.E., Baykara, T., 2000. Comparison of in vitro dissolution profiles by ANOVA-based, model-dependent and -independent methods. International Journal of Pharmaceutics 209, 57-67. 
663 Zhang, Y., Huo, M., Zhou, J., Zou, A., Li, W., Yao, C., Xie, S., 2010. DDSolver: An Add-In Program for 664 Modeling and Comparison of Drug Dissolution Profiles. The AAPS Journal 12, 263-271.

665 Zhao, M., Barker, S.A., Belton, P.S., McGregor, C., Craig, D.Q.M., 2012. Development of fully

666 amorphous dispersions of a low Tg drug via co-spray drying with hydrophilic polymers. European

667 Journal of Pharmaceutics and Biopharmaceutics 82, 572-579.

668

669 
Table 1. Cocrystal formation in excipient matrix when spray dried at a ratio of 50:50

673 (w/w) cocrystal components: excipient. The calculated HSP of SDM:4ASA cocrystal was $67426.8 \mathrm{MPa}^{0.5}$. Key, CC, cocrystal.

\begin{tabular}{|c|c|c|c|c|}
\hline Excipient & $\begin{array}{c}\text { Crystalline or } \\
\text { amorphous nature } \\
\text { of the excipient }\end{array}$ & $\begin{array}{c}\mathrm{\delta}_{\mathrm{t}}\left(\mathrm{MPa}^{0.5}\right) \text { of } \\
\text { excipient } \\
\text { (Reference) }\end{array}$ & $\begin{array}{c}\Delta \delta_{\mathrm{t}}\left(\mathrm{MPa}^{0.5}\right) \\
\text { between excipient } \\
\text { and Cocrystal } \\
\end{array}$ & $\begin{array}{l}\text { PXRD of co-spray } \\
\text { dried systems }\end{array}$ \\
\hline Inulin & Amorphous & 45.4 & 18.6 & $\mathrm{CC}$ \\
\hline MCC & Amorphous & 39.3 (Rowe, 1988) & 12.5 & $\mathrm{CC}$ \\
\hline Mannitol & Crystalline & $\begin{array}{c}39.1 \text { (Forster et al., } \\
2001 \text { ) }\end{array}$ & 12.3 & $\mathrm{CC}$ \\
\hline Chitosan & Amorphous & $\begin{array}{c}38 \text { (Ravindra et al., } \\
\text { 1998) }\end{array}$ & 11.2 & $\mathrm{CC}+\mathrm{API}+$ coformer \\
\hline Dextran & Amorphous & $\begin{array}{l}36.4 \text { (Antoniou et } \\
\text { al., 2010) }\end{array}$ & 9.6 & $\mathrm{CC}$ \\
\hline Glycine & Crystalline & 33.4 & 6.6 & $\mathrm{CC}+\mathrm{API}+$ coformer \\
\hline PVA & Amorphous & $\begin{array}{c}31.7 \text { (Forster et al., } \\
2001 \text { ) }\end{array}$ & 4.9 & $\mathrm{CC}+\mathrm{API}+$ coformer \\
\hline HPMC & Amorphous & 28.7 & 1.9 & Amorphous \\
\hline PVP & Amorphous & $\begin{array}{c}22.4 \text { (Forster et al., } \\
\text { 2001) }\end{array}$ & 4.4 & Amorphous \\
\hline Soluplus & Amorphous & 22.9 & 3.9 & Amorphous \\
\hline
\end{tabular}

675

676 
678 Table 2. Solubility values of cocrystal and individual components in excipients and the 679 associated difference in HSP.

\begin{tabular}{|l|l|l|}
\hline System & Solubility (\%w/w) & Difference in HSP (MPa \\
\hline Cocrystal in Inulin & 3.69 & 18.6 \\
\hline Cocrystal in MCC & 3.85 & 12.5 \\
\hline Cocrystal in Chitosan & 3.23 & 11.2 \\
\hline Cocrystal in Dextran & 3.83 & 9.6 \\
\hline Cocrystal in PVA & 13.74 & 4.9 \\
\hline Cocrystal in PVP & 24.43 & 4.4 \\
\hline Cocrystal in Soluplus & 25.21 & 3.9 \\
\hline Cocrystal in HPMC & 18.77 & 1.9 \\
\hline SDM in Inulin & 2.85 & 19.2 \\
\hline 4ASA in Inulin & 4.14 & 16.8 \\
\hline 4ASA in MCC & 1.77 & 10.7 \\
\hline SDM in Chitosan & 2.50 & 11.8 \\
\hline 4ASA in Chitosan & 9.41 & 9.4 \\
\hline SDM in Dextran & 5.68 & 10.2 \\
\hline 4ASA in Dextran & 5.10 & 7.8 \\
\hline SDM in PVA & 13.88 & 5.5 \\
\hline 4ASA in PVA & 11.77 & 3.1 \\
\hline SDM in Soluplus & 15.93 & 3.3 \\
\hline 4ASA in PVP & 27.52 & \\
\hline
\end{tabular}

680

Table 3. Intrinsic dissolution rates of SDM calculated over the first $10 \mathrm{~min}$.

\begin{tabular}{|c|c|}
\hline System, 50:50, w:w ratio & Initial Dissolution Rate $\left(\mathrm{mg} / \mathrm{cm}^{2} / \mathrm{min}\right)$ \\
\hline Cocrystal in inulin system & $0.0712 \pm 0.0027$ \\
\hline Cocrystal in mannitol system & $0.0812 \pm 0.0013$ \\
\hline Cocrystal in dextran system & $0.0764 \pm 0.0150$ \\
\hline
\end{tabular}


686 Table 4. Prediction of cocrystal formation based on calculated CFP values (from Eq. 6). 687 Darker areas $(\mathrm{CFP}<1)$ indicate that the formation of a co-amorphous system is likely, while 688 lighter areas $(\mathrm{CFP}>10)$ indicate that there is a high likelihood of cocrystal formation to occur 689 in the co-spray dried system.

\begin{tabular}{cccccccccc}
\hline & \multicolumn{7}{c}{ Ratio of Excipient } \\
Excipient & 0.1 & 0.2 & 0.3 & 0.4 & 0.5 & 0.6 & 0.7 & 0.8 & 0.9 \\
\hline Inulin & 50.1 & 25.1 & 16.7 & 12.5 & 10 & 8.4 & 7.2 & 6.3 & 5.6 \\
MCC & 30.5 & 15.3 & 10.2 & 8 & 6.1 & 5.1 & 4.4 & 3.8 & 3.4 \\
Chitosan & 34.4 & 17.2 & 11.5 & 8.6 & 6.9 & 5.7 & 4.9 & 4.3 & 3.8 \\
Dextran & 24.8 & 12.4 & 8.3 & 6.2 & 5 & 4.1 & 3.5 & 3.1 & 2.8 \\
PVA & 3.5 & 1.7 & 1.2 & 0.9 & 0.7 & 0.6 & 0.5 & 0.4 & 0.4 \\
PVP & 1.6 & 0.8 & 0.5 & 0.4 & 0.3 & 0.3 & 0.2 & 0.2 & 0.2 \\
Soluplus & 1.5 & 0.8 & 0.5 & 0.4 & 0.3 & 0.3 & 0.2 & 0.2 & 0.2 \\
HPMC & 1 & 0.5 & 0.3 & 0.2 & 0.2 & 0.2 & 0.1 & 0.1 & 0.1 \\
\hline
\end{tabular}

690

691 


\section{Production of Cocrystals in an Excipient Matrix by Spray Drying}

David Walsh $^{\mathrm{a}, \mathrm{b}}$, Dolores R. Serrano ${ }^{\mathrm{a}, \mathrm{b}}$, Zelalem Ayenew Worku ${ }^{\mathrm{a}, \mathrm{b}}$, Brid A. Norris ${ }^{\mathrm{a}, \mathrm{b}}$, Anne Marie Healy ${ }^{\mathrm{a}, \mathrm{b}^{*}}$

${ }^{\mathrm{a}}$ School of Pharmacy and Pharmaceutical Sciences, Trinity College Dublin, Dublin 2, Ireland.

${ }^{\mathrm{b}} \mathrm{SSPC}$, Synthesis and Solid State Pharmaceutical Centre, Ireland.

*Corresponding author:

Tel.: +35318961444

E-mail address: healyam@tcd.ie (A.M. Healy) 
Figure 1. PXRD patterns and DSC thermograms of cocrystals and co-spray dried systems. i) PXRD patterns a) Cocrystal produced by spray drying, b) Cocrystal produced by slow solvent evaporation from acetone, c) Unprocessed 4ASA, d) Unprocessed SDM. ii) PXRD pattern of co-spray dried systems with excipient at $50 \% \mathrm{w} / \mathrm{w}$ ratio. a) Cocrystal produced by spray drying, b) Cocrystal components co-spray dried with dextran, c) Cocrystal components co-spray dried with inulin, d) Cocrystal components co-spray dried with MCC, e) Cocrystal components co-spray dried with mannitol. iii) DSC thermograms. a) Unprocessed SDM, b) Unprocessed 4ASA, c) Cocrystal produced by spray drying, d) Cocrystal produced by solvent evaporation, e) Cocrystal components co-spray dried with inulin, f) Cocrystal components co-spray dried with mannitol, g) Cocrystal components cospray dried with MCC, h) Cocrystal components co-spray dried with dextran.

Figure 2. PXRD patterns of co-spray dried systems with soluplus and PVP. i) Co-spray dried with Soluplus and ii) Co-spray dried with Soluplus after stressing at $25{ }^{\circ} \mathrm{C}$ and $60 \% \mathrm{RH}$ for seven days. a) Spray dried cocrystal, b) Cocrystal:soluplus (75:25, w:w), c) Cocrystal:soluplus (80:20, w:w), d) Cocrystal:soluplus (90:10, w:w). iii) Co-spray dried with PVP and iv) Co-spray dried with PVP after stressing at $25^{\circ} \mathrm{C}$ and $60 \% \mathrm{RH}$ for seven days, a) Spray dried cocrystal, b) Cocrystal:PVP (75:25, w:w), c) Cocrystal:PVP (80:20, w:w), d) Cocrystal:PVP (90:10, w:w).

Figure 3. DSC thermograms (i) and PXRD pattern (ii) of co-spray dried cocrystal with chitosan. Key: i) a) Spray dried cocrystal, b) Unprocessed SDM, c) Unprocessed 4ASA, d) Cocrystal:Chitosan (75:25, w:w), e) Cocrystal:Chitosan (80:20, w:w), f) Cocrystal Cocrystal:Chitosan (90:10, w:w). ii) a) Spray dried cocrystal, b) Cocrystal:Chitosan (70:30, w:w), c) Cocrystal:Chitosan (75:25, w:w), d) Cocrystal:Chitosan (80:20, w:w), e) Cocrystal:Chitosan (90:10, w:w).

Figure 4. PXRD patterns of co-spray dried systems with MCC before (i) and after stressing (ii) at $25^{\circ} \mathrm{C}$ and $60 \%$ RH for seven days. Key: a) Spray dried cocrystal, b) Cocrystal:MCC (50:50, w:w), c) Cocrystal:MCC (40:60, w:w), d) Cocrystal:MCC (30:70, w:w), e) Cocrystal:MCC (20:80, w:w), f) Unprocessed MCC. iii) DSC thermograms of cospray dried systems with MCC. Key: a) Spray dried cocrystal, b) Cocrystal:MCC (50:50, w:w), c) Cocrystal:MCC (40:60, w:w), d) Cocrystal:MCC (30:70, w:w), e) Cocrystal:MCC (20:80, w:w). 
Figure 5. SEM micrographs. Key: a) Spray dried cocrystal, b) Co-spray dried cocrystal with inulin $(50: 50, \mathrm{w}: \mathrm{w}), \mathrm{c})$ Co-spray dried cocrystal with mannitol $(50: 50, \mathrm{w}: \mathrm{w})$, d) Co-spray dried cocrystal with MCC (50:50, w:w), e) Co-spray dried cocrystal with PVP (50:50, w:w), f) Co-spray dried cocrystal with PVP (90:10, w:w).

Figure 6. FTIR analyses of a) co-spray dried cocrystal in inulin (50:50, w/w ratio), b) spray dried cocrystal, c) inulin, d) a physical mixture of SDM and 4ASA (1:1 molar ratio).

Figure 7. The solubility of the cocrystal in inulin (i), MCC (ii), chitosan (iii) and dextran (iv).

Figure 8. The solubility of the cocrystal in PVA (i), Soluplus (ii), HPMC (iii) and PVP (iv).

Figure 9. The release of SDM for the systems co-spray dried with inulin (black $\square$ ), mannitol (red $\bullet$ ) and dextran (blue $\Delta$ ) with a 50:50\% w/w ratio of excipient and cocrystal.

Figure 10. Tensile strength (circles) and ejection force (triangles) of i) co-spray dried system and physical mixtures of cocrystal 50\%, MCC 50\%, and ii) co-spray dried system and physical mixtures of cocrystal $60 \%$, inulin $20 \%$ and MCC $20 \%$, compacted at $6 \mathrm{KN}$. 


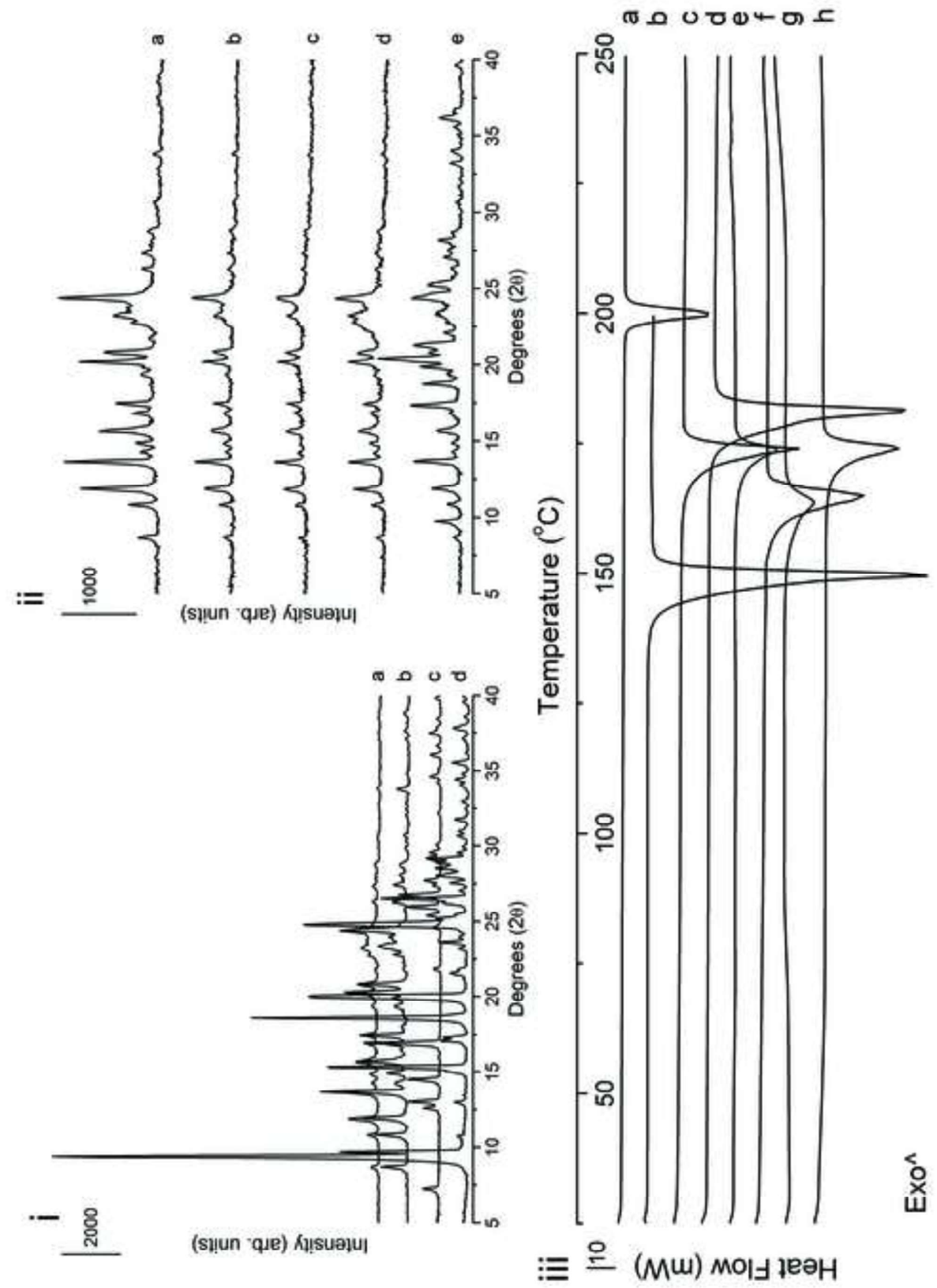



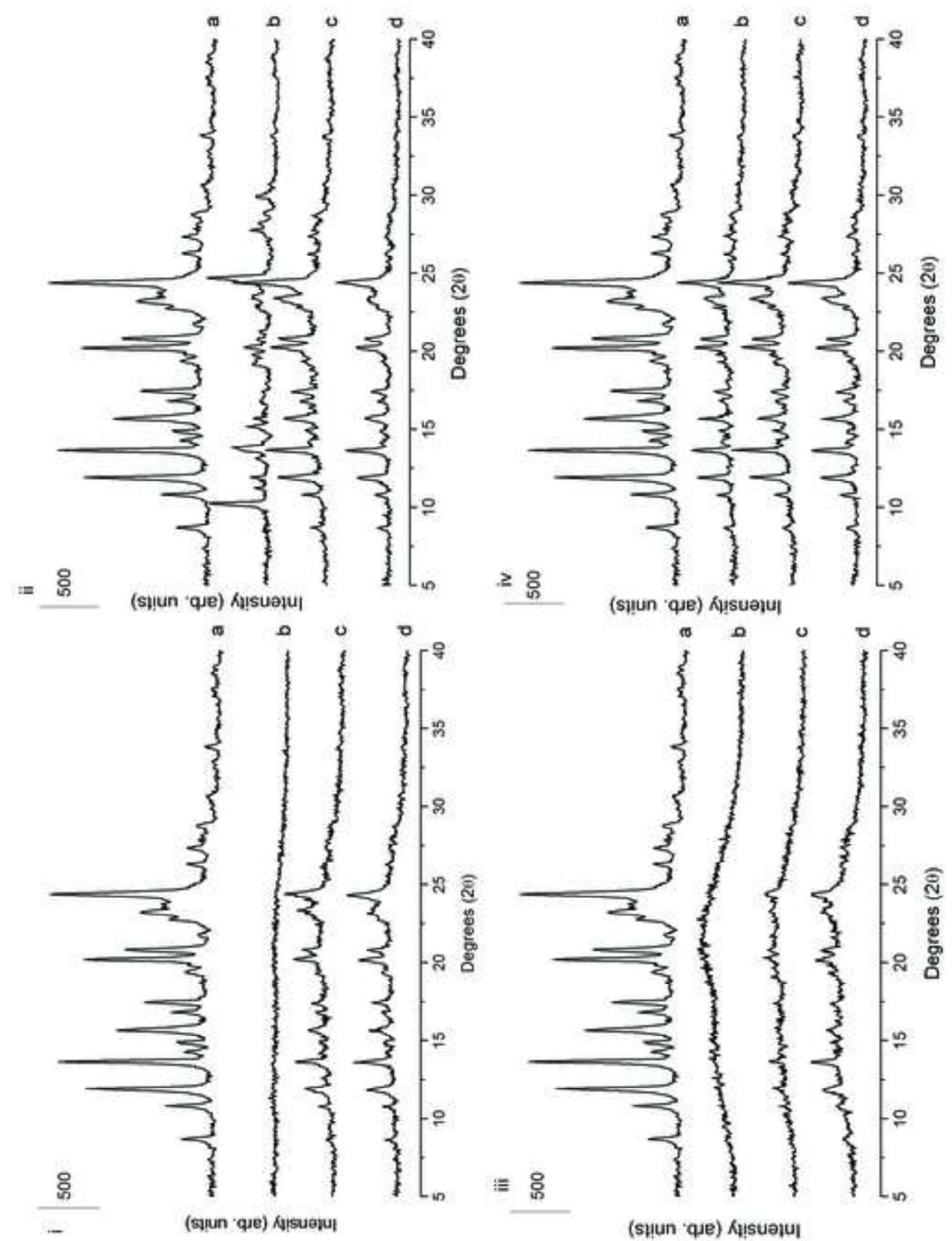

(shun 'qu) Kı̣suə̣u| 


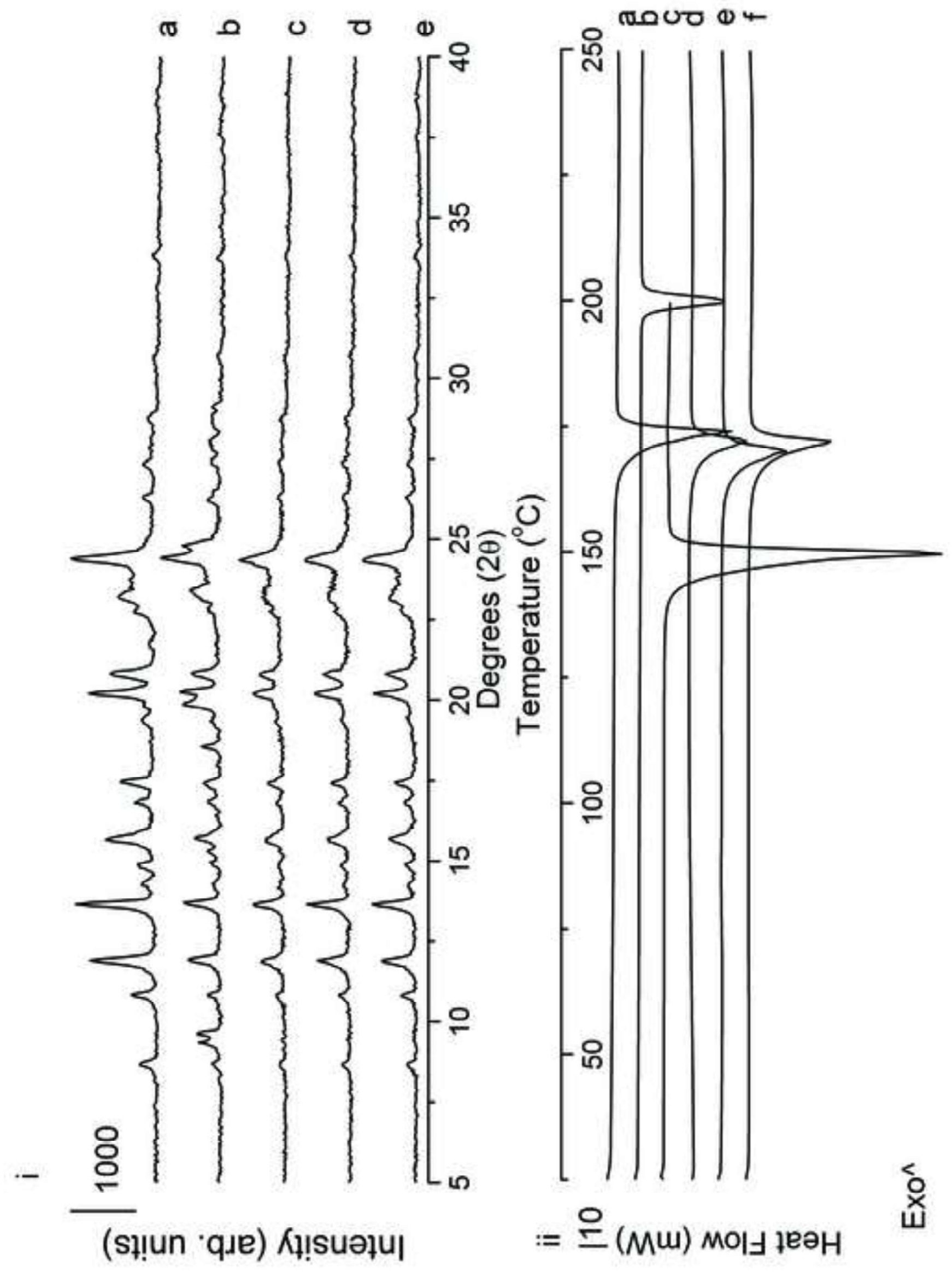

인 


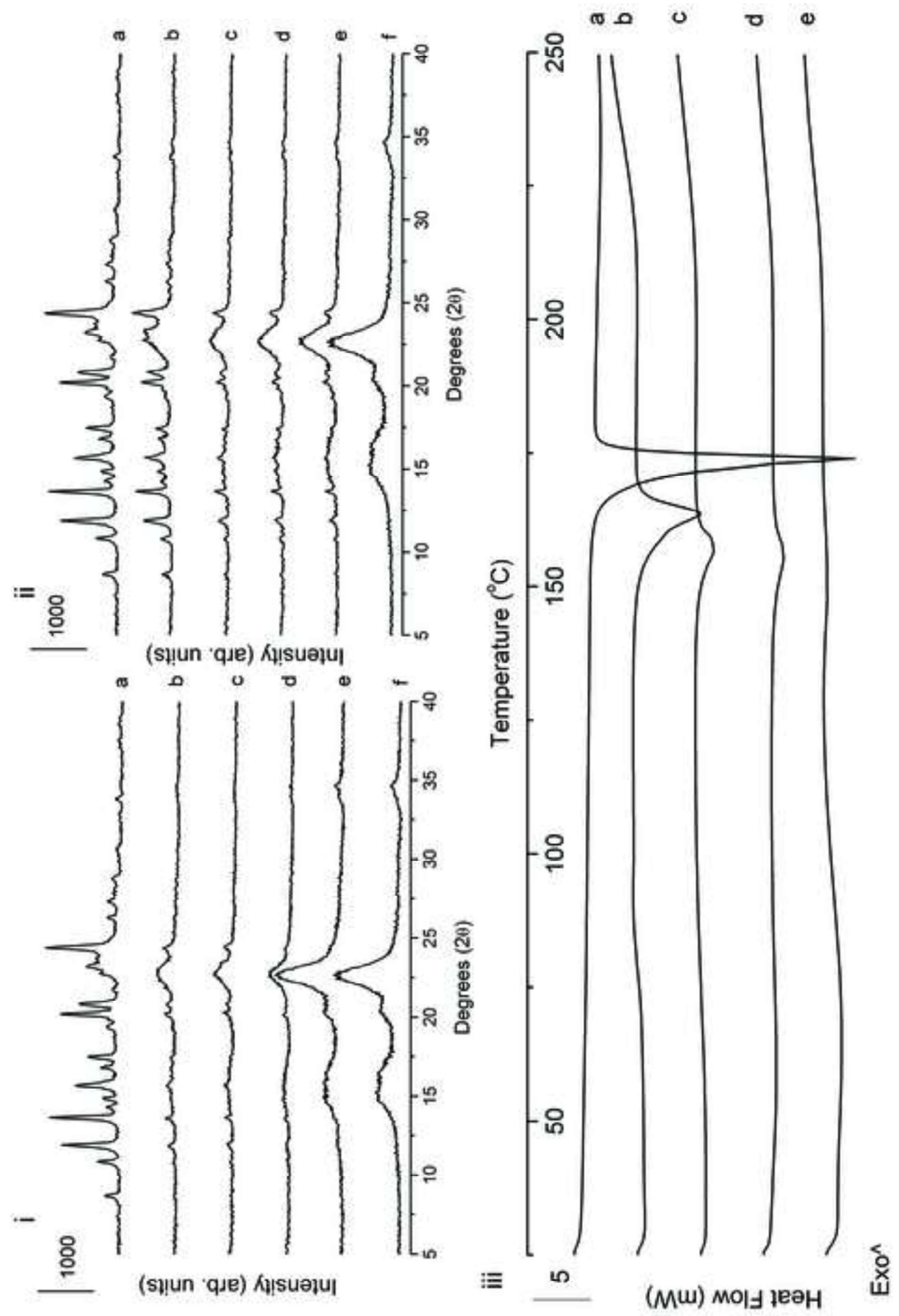

寸
은
운 


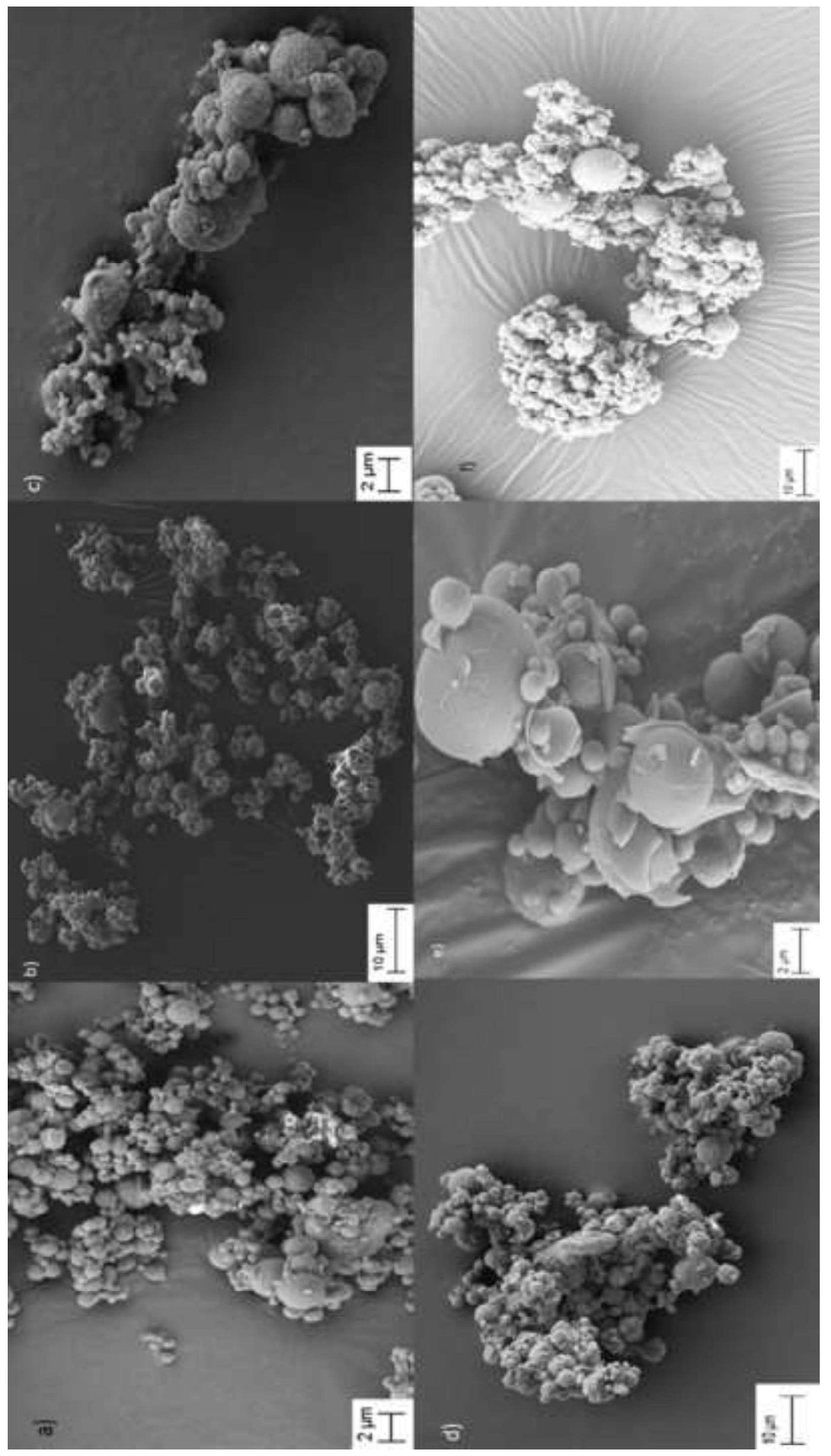




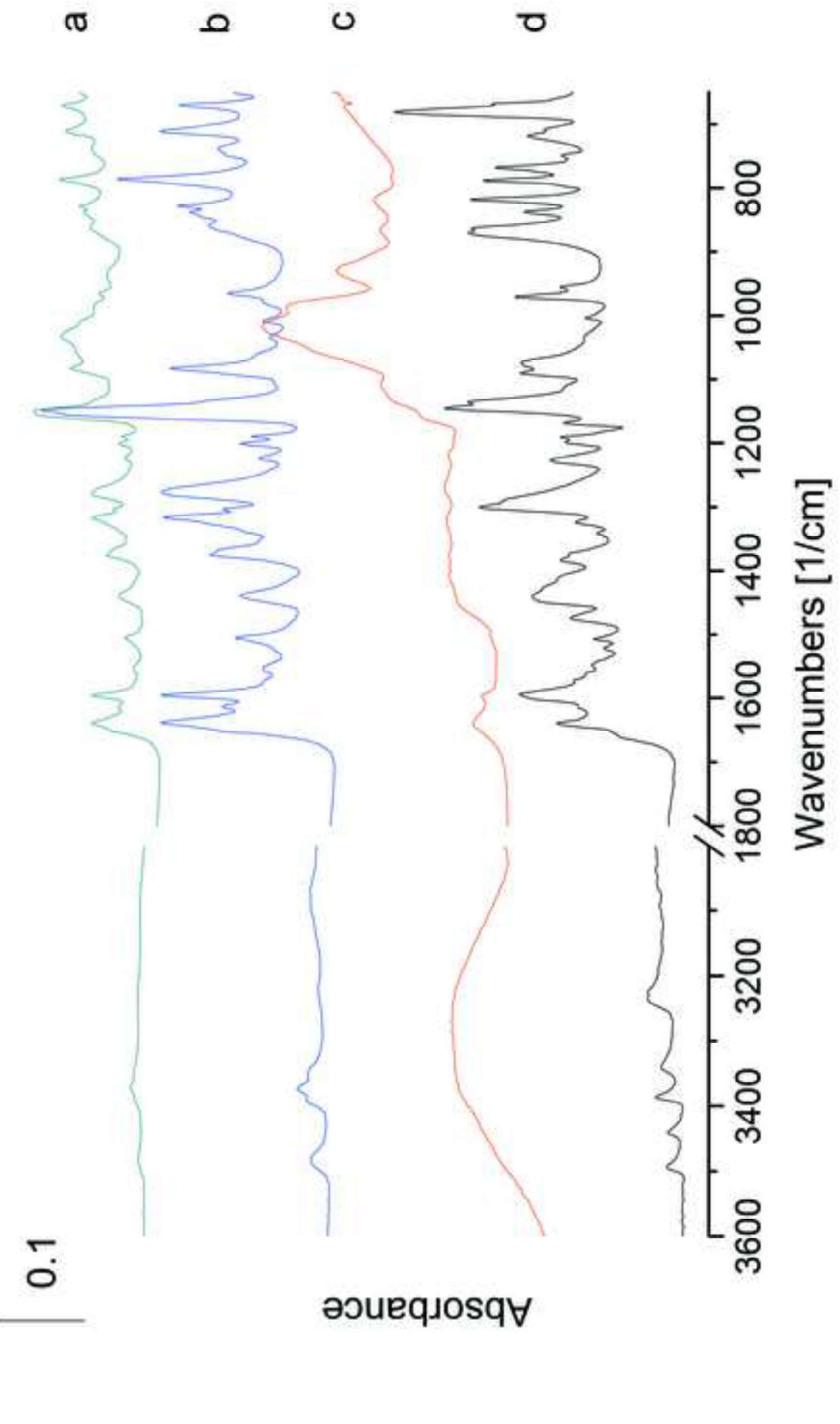




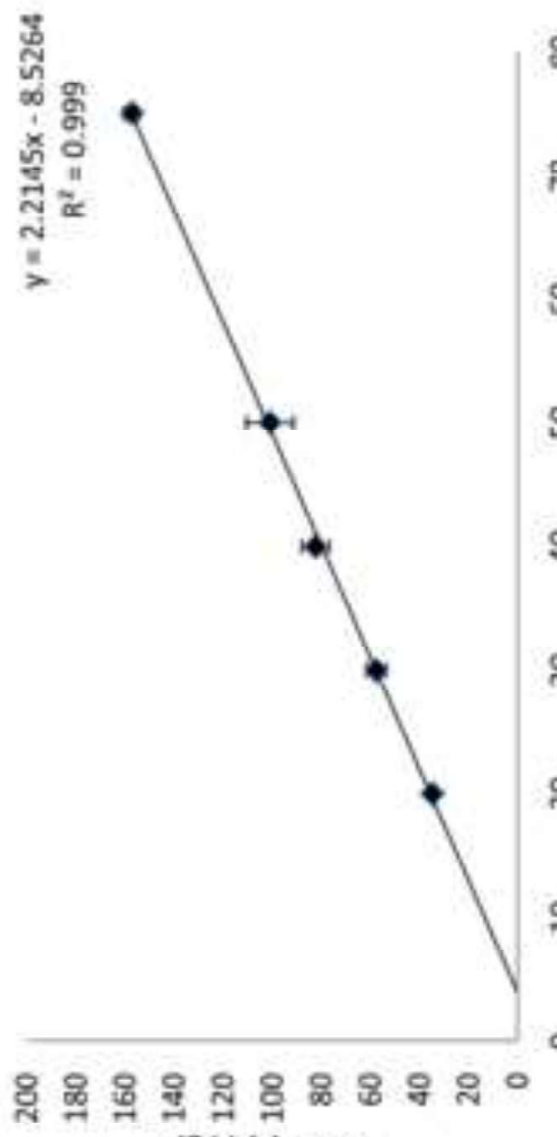

$$
:=\quad(8 / f) \text { Adjeyzuz }
$$

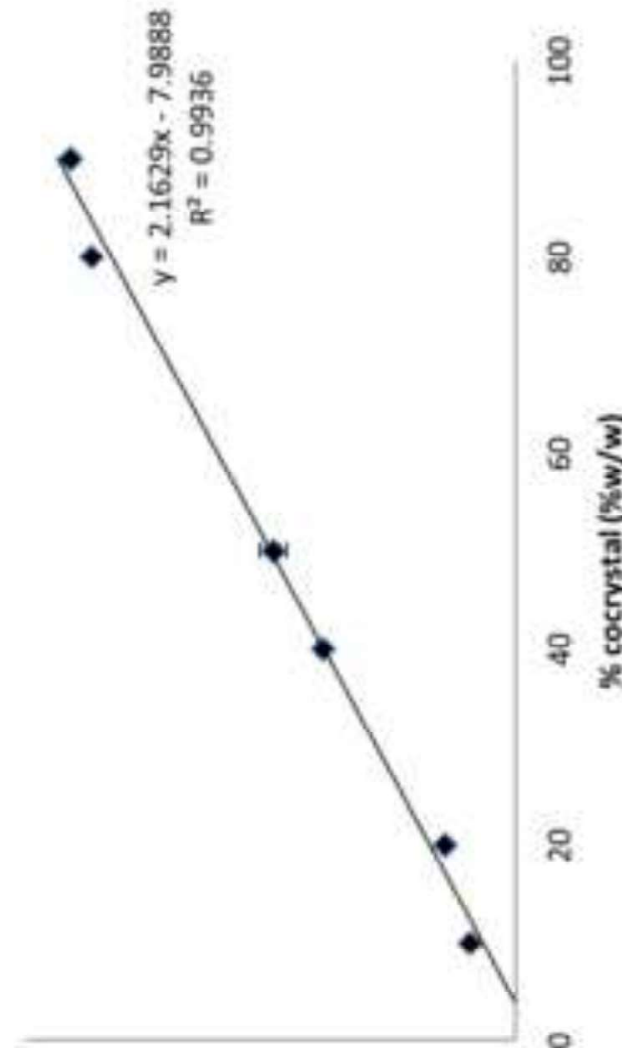

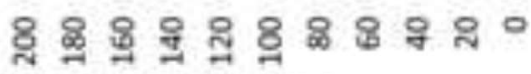

(3/f) Adjeypus

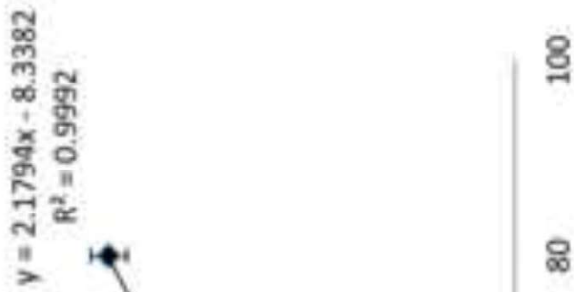

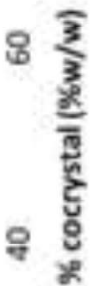

오

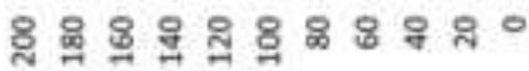
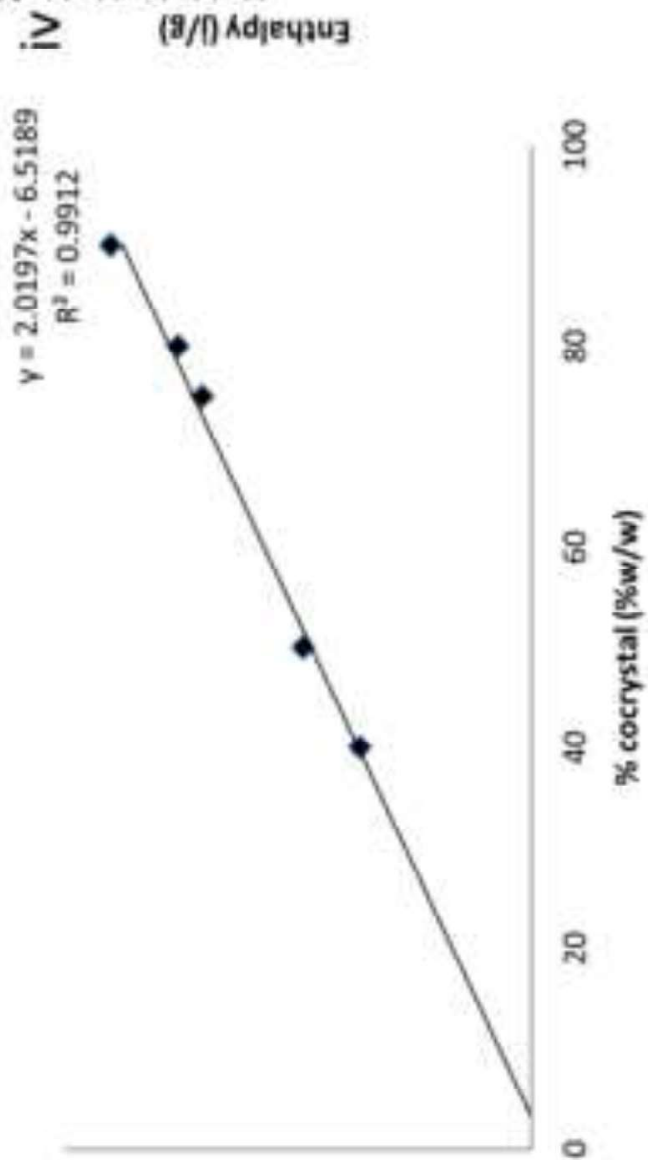

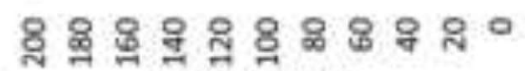

(\$//) Adpeyju 3 

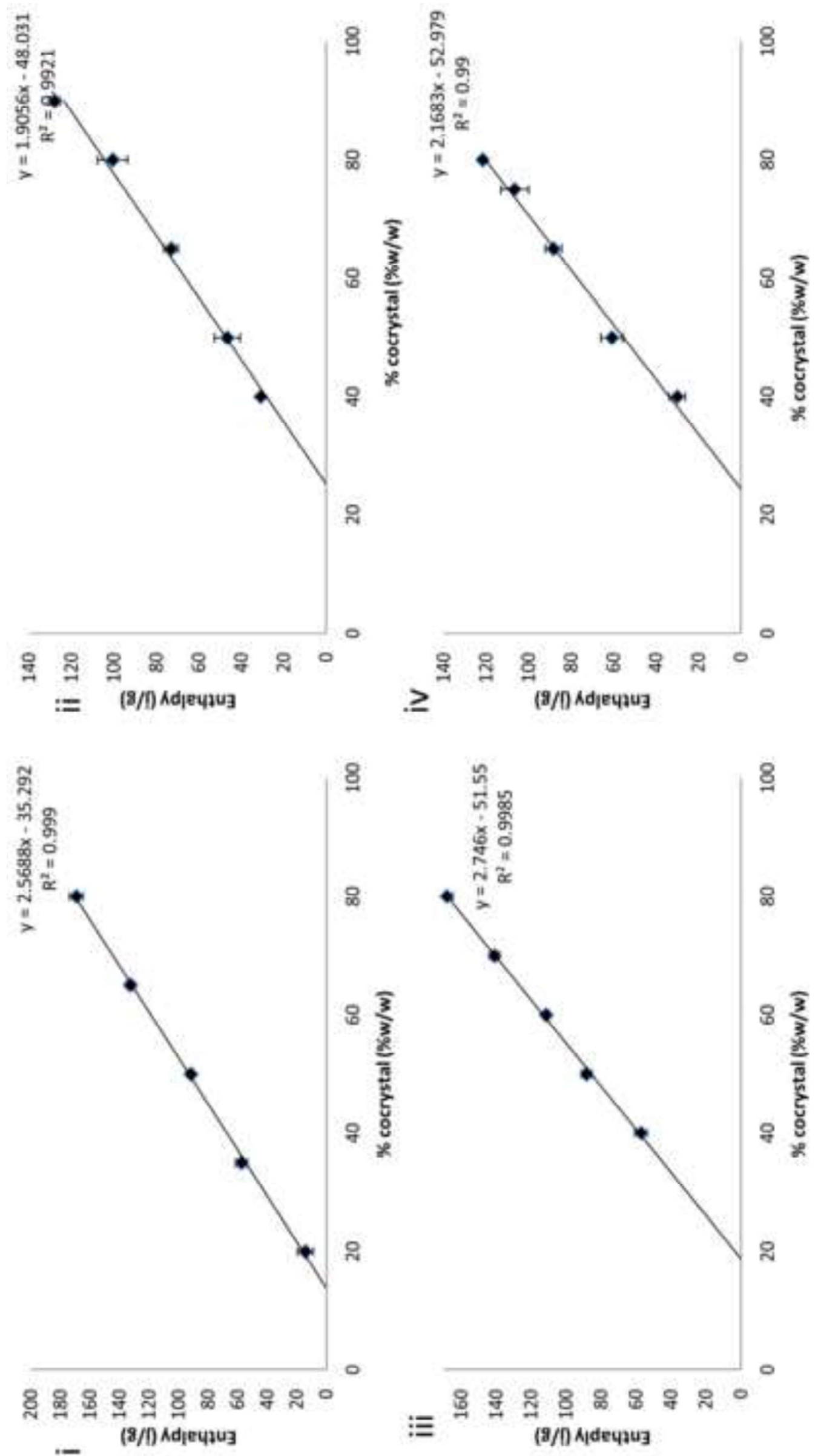

인 


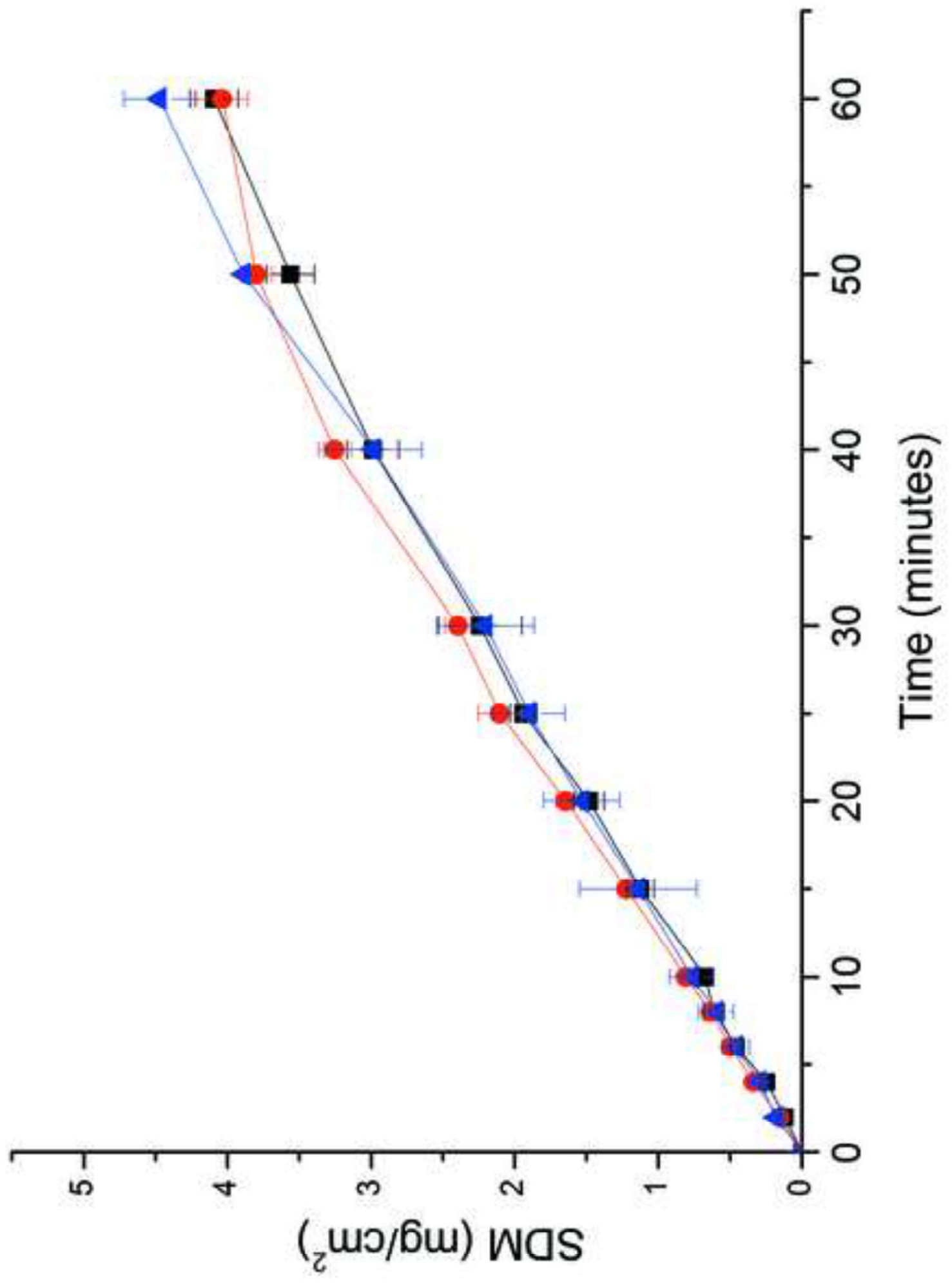

임
는
문 
Ejection force $(\mathrm{N})$

Ejection force $(\mathrm{N})$

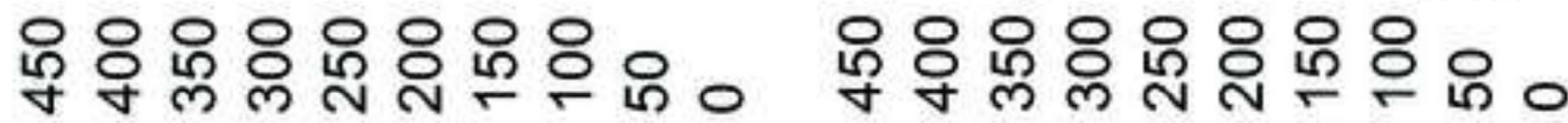

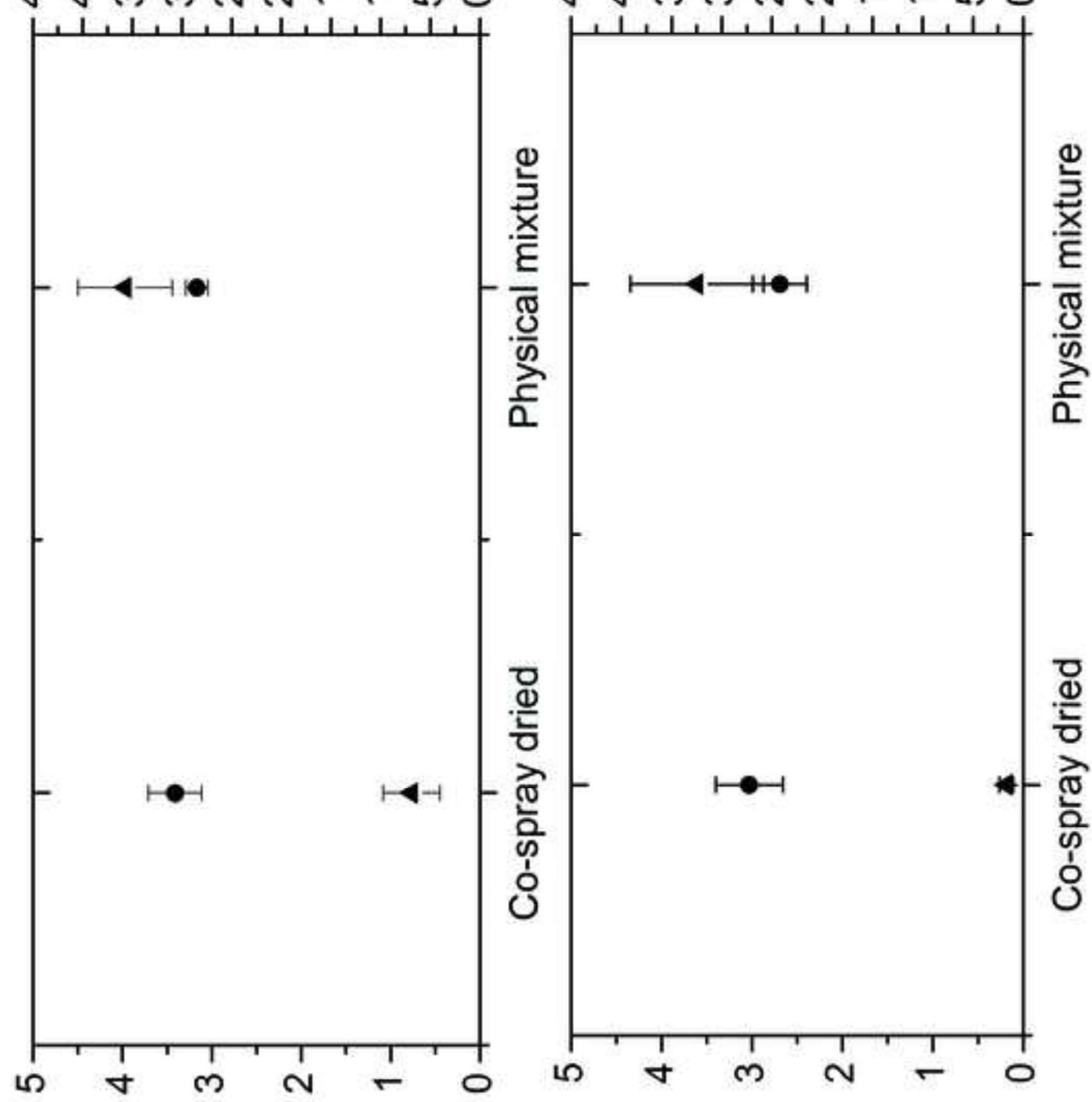

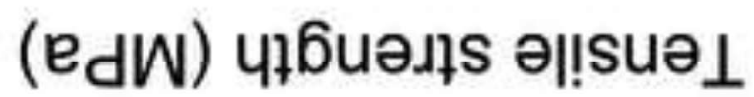

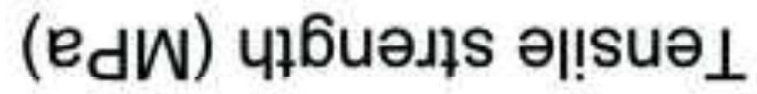

